\title{
Wall-modelled large-eddy simulation of turbulent flow past airfoils
}

\author{
Wei Gao ${ }^{1, \dagger}$, Wei Zhang ${ }^{1}$, Wan Cheng ${ }^{1}$ and Ravi Samtaney ${ }^{1}$ \\ ${ }^{1}$ Mechanical Engineering, Physical Science and Engineering Division, \\ King Abdullah University of Science and Technology, Thuwal 23955-6900, Saudi Arabia
}

(Received 23 November 2018; revised 21 March 2019; accepted 28 April 2019; first published online 24 June 2019)

We present large-eddy simulation (LES) of flow past different airfoils with $R e_{c}$, based on the free-stream velocity and airfoil chord length, ranging from $10^{4}$ to $2.1 \times 10^{6}$. To avoid the challenging resolution requirements of the near-wall region, we develop a virtual wall model in generalized curvilinear coordinates and incorporate the non-equilibrium effects via proper treatment of the momentum equations. It is demonstrated that the wall model dynamically captures the instantaneous skin-friction vector field on arbitrary curved surfaces at the resolved scale. By combining the present wall model with the stretched-vortex subgrid-scale model, we apply the wall-modelled LES approach to three different airfoil cases, spanning different geometrical parameters, different attack angles and low to high $R e_{c}$. The numerical results are verified with direct numerical simulation (DNS) at low $R e_{c}$, and validated with experiment data at higher $R e_{c}$, including typical aerodynamic properties such as pressure coefficient distributions, velocity components and also more challenging measurements such as skin-friction coefficient and Reynolds stresses. All comparisons show reasonable agreement, providing a measure of validity that enables us to further probe simulation results into aspects of flow physics that are not available from experiments. Two techniques to quantify hitherto unexplored physics of flows past airfoils are employed: one is the construction of the anisotropy invariant map, and the second is skin-friction portraits with emphasis on flow transition and unsteady separation along the airfoil surface. The anisotropy maps for all three $R_{c}$ cases, show clearly that a portion of the flow field is aligned along the axisymmetric expansion line, corresponding to the turbulent boundary layer log-law behaviour and the appearance of turbulent transition. The instantaneous skin-friction portraits reveal a monotonic shrinking of the near wall structure scale. At $R e_{c}=10^{4}$, the interaction between the primary separation bubble and the secondary separation bubble contributes to turbulent transition, similar to the case of flow past a cylinder. At higher $R e_{c}=10^{5}$, the primary separation breaks into several small separation bubbles. At even higher $R e_{c}=2.1 \times 10^{6}$, near the turbulent separation, the skin-friction lines show small-scale reversal flows that are similar to those observed in DNS of the flat plate turbulent separation. A notable feature of turbulent separation in flow past an airfoil is the appearance of turbulence structures and small-scale reversal flows in the spanwise direction due to the vortex shedding behaviour.

Key words: boundary layer separation, turbulence simulation 


\section{Introduction}

External flow past an isolated airfoil is relevant in the context of a variety of engineering applications, such as micro or unmanned air vehicles, small wind turbines and low-speed aircraft. Key aerodynamic quantities such as the lift and drag coefficients, of relevance to engineering applications, have been the focus of several experimental investigations (Abbott, Doenhoff \& Stivers 1945; Lissaman 1983; Laitone 1997) at different Reynolds numbers $\left(R e_{c}\right)$ and angles of attack $(A o A)$. Recently, a growing body of experimental studies focusing on the boundary layer separation and transition process have been reported (Nakano, Fujisawa \& Lee 2006; Yarusevych, Sullivan \& Kawall 2006, 2009; Boutilier \& Yarusevych 2012). It is found that the complex flow patterns (i.e. separation, transition and reattachment) on the suction side deteriorate aerodynamic performance by negatively affecting airfoil lift and drag (Yarusevych et al. 2009). These studies imply that a detailed analysis of the unsteady flow dynamics is required for further shape optimization and flow control.

High Reynolds unsteady flow past an airfoil is characterized by boundary layer separation, laminar-turbulent transition, wall-bounded turbulence under pressure gradients and turbulent wake flow. Methods to simulate this full three-dimensional (3-D) unsteady flow include: direct numerical simulation (DNS), large-eddy simulation (LES), detached-eddy simulation (DES) and Reynolds-averaged Navier-Stokes models (RANS). All the near-wall turbulent scales are resolved in DNS, requiring mesh resolution that scales as $R e^{37 / 14}$ (Choi \& Moin 2012): this restriction on resolution proves to be untenable for high $R e$ wall-bounded flows. Recently, Hosseini et al. (2016) performed DNS of flow past a NACA4412 airfoil at a Reynolds number based on free-stream velocity $U_{\infty}$ and chord length $C$ of $R e_{c}=4 \times 10^{5}$. To the best of our knowledge, this is by far the highest $R e_{c}$ airflow case simulated with DNS. A viable alternative to DNS is LES in which the large scales are resolved while the effects of small scales are modelled using a subgrid-scale (SGS) model. LES of wall-bounded turbulent flows can be classified into wall-resolved large-eddy simulations (WRLES) and wall-modelled large-eddy simulations (WMLES). In wall-bounded turbulent flows, the near-wall eddies scale with wall units, imposing a significant computational cost to sufficiently resolve them in practical simulations. The near-wall resolution problem is exacerbated for high $R e$ turbulent flows. Choi \& Moin (2012) estimated that the number of mesh points for WRLES is $O\left(R e^{13 / 7}\right)$, while for WMLES, the mesh points requirement scales linearly with increasingly $R e$, i.e. $N_{w m} \sim O(R e)$, where $N_{w m}$ is the number of mesh points needed in WMLES. Hence, the wall-modelling approach is a tenable solution for LES of high $R e$ wall-bounded turbulent flows.

In the past four decades, several wall models have been proposed for canonical flows in simple geometries (Schumann 1975; Grötzbach 1987; Piomelli et al. 1989; Marusic, Kunkel \& Porté-Agel 2001; Piomelli \& Balaras 2002). However, there are a couple of primary challenges when it comes to practical engineering simulations. First, most wall models follow the equilibrium stress assumption and imply a log-law profile in the near-wall region, which is a questionable assumption for turbulent boundary layers subjected to strong adverse pressure gradients (APG) leading to separation, extra strains due to curvature, etc. (Diurno 2001). Second, most wall-modelling strategies including DES fall into the hybrid RANS/LES methodology in complex geometries, which solves the simplified or full RANS equations in the inner layer and provides wall shear stress boundary conditions for the outer LES region (Cabot \& Moin 1999; Piomelli \& Balaras 2002; Kawai \& Asada 2013; Park \& Moin 2016). This hybrid method is not only sensitive to the choice of the RANS model and its associated model coefficients, but also leads to the so-called 'scale disparity' problem 
on the nominal interfaces between the RANS and LES regions (Germano 2004; Piomelli 2008). Recently, Bose \& Moin (2014) proposed a differential filter-based wall model with a specific choice of the filter kernel, in which a local slip length parameter is introduced and computed via a dynamic procedure. This differential model offers the Robin (slip) boundary condition, and has been tested in both canonical flows and NACA4412 airflows (Bose \& Moin 2014; Bae et al. 2019). Although no a priori coefficients are specified, the accuracy of the slip wall model is not only limited by the robustness of the dynamic procedure to compute the slip length, but also sensitive to the SGS models and numerical methods (Bose \& Park 2018).

An alternative to the hybrid RANS/LES approach is the virtual wall model proposed by Chung \& Pullin (2009). In this approach one dynamically couples the outer resolved LES region with the inner wall region, and offers a slip velocity boundary condition for the filtered LES velocity field on the 'virtual wall'. This wall model was successfully applied in canonical flows without separation (Inoue \& Pullin 2011; Saito, Pullin \& Inoue 2012), and then extended by Cheng, Pullin \& Samtaney (2015) to simulate the flat-plate turbulent boundary layer flows with separation and reattachment. Although the above wall models developed by Pullin and co-authors have been shown to successfully predict flat-plate wall-bounded flows and related phenomena, a missing part, which plays a key role in aerodynamical applications, is the effect of curvature due to the local geometry, and corresponding effects such as pressure gradient and turbulent transition. Development of a systematic framework for wall models of turbulent flows around extruded two-dimensional (2-D) or 3-D bodies with arbitrary geometry, would constitute an essential contribution to turbulent flow simulations, paving the way for WMLES for more realistic aerodynamic applications. Indeed, the present study is a starting point for the development of a general WMLES framework.

In the present investigation, we emphasize two main objectives. One of the main objectives of this work is to extend the virtual wall model to the generalized curvilinear coordinates. Since we incorporate both momentum equations for both wall-parallel directions, the present general wall model naturally possesses the ability to handle most of the significant turbulent flow features along a curved surface, not only the wall attached flows with either a zero pressure gradient (ZPG) or varying pressure gradient, but also the separation/reattachment and its related phenomena such as turbulent transition on the top side of a separation bubble. The present implementation is on body-fitted structured meshes that are commonly employed for complex geometries. We emphasize strong validation of our WMLES results. By 'strong' we mean going beyond the comparisons of pressure coefficient, and lift and drag coefficients and present detailed comparisons of our results with experiments of skin-friction coefficient and Reynolds stresses wherever available.

Another major objective of this work is to examine the details of unsteady separation for turbulent flow past airfoils. Recent work by Cheng et al. (2017), Cheng, Pullin \& Samtaney (2018a) in WRLES of flow past a smooth and grooved cylinder at subcritical and supercritical Reynolds numbers, emphasized the role of unsteady separation and the dynamics of separation bubbles in the phenomenon of the drag crisis. Their explanation is that the drag crisis, mainly due to a large change in form drag, is due to the topology change induced by the movement of the location of curvature-controlled large-scale separation. Furthermore, although near-wall shear-layer turbulent transition is observed in flow over a smooth cylinder, it does not occur in flow past a grooved cylinder. In the present study, we note the 
airfoil geometry may be considered as a combination of large curvature (near the front stagnation part) and small curvature near the trailing edge portion of the airfoil. This geometric complexity, accompanied by different AoA, will no doubt result in rich flow physics, especially insofar as separation and transition are concerned. We employ several diagnostic tools, including instantaneous surface skin-friction lines and invariant maps of anisotropy, to reveal the flow patterns due to dynamic interaction of local separation and transition. These flow analyses aim to provide a clear physical flow description of high Reynolds number airfoil flow up to $R e_{c}=2.1 \times 10^{6}$, in ways that has not been fully examined in past several decades. In addition, we place a strong emphasis on validating our results against previous experiments.

The paper is organized as follows: in $\S 2$, we provide a brief summary on the LES of flow past airfoils, analysing the available datasets in cited references, and emphasize the important flow properties examined in the present study. In $\S 3$, the general formulation of the wall model for arbitrary curvilinear coordinate system is developed, with some details relegated to appendices A and B. Following the wall model formulation, we briefly explain the SGS model and the numerical methods employed for implementation of the wall model in LES of flow over airfoils. Large-eddy simulation results are summarized in the next several sections. In $\S 5$, the proposed model is verified against corresponding DNS (for case at $R e_{c}=10^{4}$ ), followed by detailed validation by comparing time- and spanwise-averaged quantities against experiments (for higher Reynolds number cases at $R e_{c}=10^{5}$ and $2.1 \times 10^{6}$ ). In $\S 6$, we analyse the anisotropy of the near-wall flow based on the time- and spanwise-averaged Reynolds stresses. This part provides a clear physics of the flow pattern transition around the airfoil. Later in $\S 7$, instantaneous flow field in the form of surface streamlines is analysed, with emphasis on local and large-scale separation, and also related turbulent transition behaviour. Some conclusions are finally drawn in $\S 8$.

\section{LES of flow past an airfoil: background}

To facilitate the presentation of our results, three coordinate systems are employed, as shown in figure 1: $(x, y, z)=\left(x_{1}, x_{2}, x_{3}\right)$ are Cartesian coordinates with corresponding velocity components $\left(u_{1}, u_{2}, u_{3}\right)=(u, v, w) ;(\xi, \eta, \zeta)=\left(\xi^{1}, \xi^{2}, \xi^{3}\right)$ are generalized curvilinear coordinates. The spanwise geometry is uniform in the present research, thus the $\zeta$-direction is congruent with the $z$-direction. In addition, we denote $\left(s, y_{n}, z\right)$ as coordinates with origin located at the leading edge (same as the Cartesian coordinates), where the $s$-coordinate denotes the streamwise direction (parallel to the airfoil surface), and $y_{n}$ denotes the local wall normal to the airfoil surface. The corresponding velocity components are $\left(u_{s}, u_{n}, w\right)$.

For flows past airfoils, aerodynamic quantities of interest are the integrated forces characterized by the overall lift coefficient $C_{L}$ and drag coefficient $C_{D}$; and surface quantities such as pressure coefficient scalar $C_{p}$ and surface skin friction (2-D vector on the airfoil surface) $C_{f}\left(C_{f}, C_{f_{z}}\right)$, where $C_{f}$ and $C_{f_{z}}$ denote the streamwise and spanwise skin friction coefficients, respectively. These aforementioned quantities require the flow field on the surface and near wall for computations of gradients. The off-wall flow quantities of interest include velocity vector $\boldsymbol{u}(u, v, w)$ and Reynolds stress tensor with key components $u^{\prime} u^{\prime}, v^{\prime} v^{\prime}$ and $u^{\prime} v^{\prime}$. In both numerical and experimental studies of airfoil flows, $C_{p}, C_{f}, \overline{u^{\prime} u^{\prime}}, \overline{v^{\prime} v^{\prime}}$ and $\overline{u^{\prime} v^{\prime}}$ are computed by both spanwise and time averaging (the overbar indicates averaging for Reynolds stress tensor components). We further note that available data of velocity components and Reynolds stress components may be presented in Cartesian coordinate or body-fitted coordinates. 


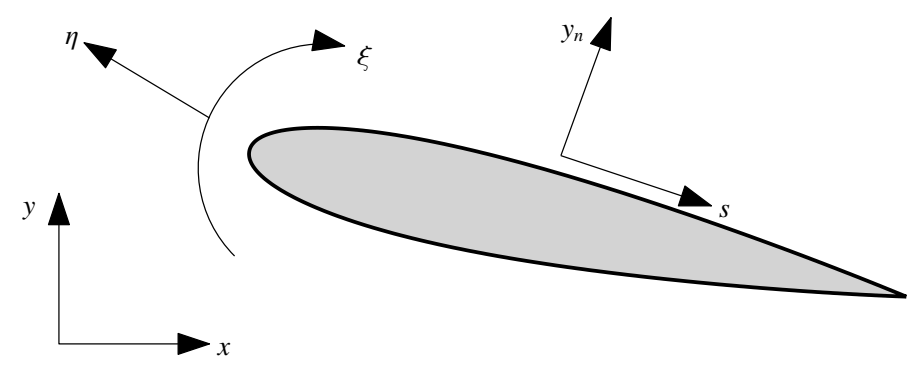

FIGURE 1. Sketch of the coordinate systems.

Author

Dahlstrom \& Davidson (2001)

Mary \& Sagaut (2002)

Morgan \& Visbal (2003)

Kawai \& Asada (2013)

Asada \& Kawai (2018)

George \& Lele (2014)

Asada et al. (2014)

Sato et al. (2016)

Schmidt, Franke \& Thiele (2001)

Bose \& Moin (2014)

Park \& Moin (2014)

Airfoil Method $\operatorname{Re}_{c} \quad A o A \quad L_{z} / C \quad$ Results

(deg.)

$\begin{array}{cclclc}\text { A-airfoil } & \text { WRLES } & 2.1 \mathrm{M} & 13.3 & 0.03 & C_{f}, C_{p}, \bar{u}, \overline{u^{\prime} v^{\prime}} \\ \text { A-airfoil } & \text { WRLES } & 2.1 \mathrm{M} & 13.3 & 0.012 & C_{f}, C_{p}, \bar{u}, \overline{u^{\prime} v^{\prime}} \\ \text { A-airfoil } & \text { WRLES } & 2.1 \mathrm{M} & 13.3 & 0.12 & C_{f}, C_{p}, \bar{u}, \overline{u^{\prime} v^{\prime}} \\ \text { A-airfoil } & \text { WMLES } & 2.1 \mathrm{M} & 13.3 & 0.017 & C_{f}, C_{p}, \bar{u}, \overline{u^{\prime} v^{\prime}} \\ \text { A-airfoil } & \text { WRLES } & 2.1 \mathrm{M} & 13.3 & 0.049 & C_{f}, C_{p}, \bar{u}, \overline{u^{\prime} v^{\prime}} \\ \text { NACA0012 } & \text { WMLES } & 1.5 \mathrm{M} & 6 & 0.14 & C_{p}, \bar{u}, \overline{u^{\prime} v^{\prime}} \\ \text { NACA0015 } & \text { WRLES } & 1.6 \mathrm{M} & 20.11 & 0.05 & C_{p}, \bar{u} \\ \text { NACA0015 } & \text { WRLES } & 1.6 \mathrm{M} & 8.13 & 0.05 & C_{L}, C_{D}, C_{p}, \bar{u} \\ \text { NACA4412 } & \text { WRLES } & 1.64 \mathrm{M} & 12 & 0.05 & C_{p}, \bar{u}, \overline{u^{\prime} v^{\prime}} \\ \text { NACA4412 } & \text { WMLES } & 1.6 \mathrm{M} & 13.8 & 0.1 & C_{p}, \bar{u} \\ \text { NACA4412 } & \text { WMLES } & 1.64 \mathrm{M} & 12 & 0.72 & C_{p}, \bar{u}\end{array}$

TABLE 1. Summary of LES performed in flow past an airfoil at high $R e_{c}$. Here suffix ' $\mathrm{M}$ ' refers to million; $\bar{u}$ denotes the velocity, $\overline{u^{\prime} v^{\prime}}$ refers to the Reynolds stress tensor components, $C_{p}$ is the pressure coefficient, $C_{f}$ is the skin-friction coefficient and $L_{z} / C$ is the ratio of the spanwise domain size to chord length.

Although many LES have been performed to investigate the flow dynamics past an airfoil at low $R e_{c}$, few results have been published at high $\operatorname{Re}_{c}\left(\operatorname{Re}_{c} \geqslant 10^{6}\right)$. Here we tabulate previous LES studies of flows past isolated airfoils for $R e_{c} \geqslant 10^{6}$ (see table 1). It is noted that the skin-friction coefficient $\left(C_{f}\right)$ and Reynolds stress $\left(\overline{u^{\prime} v^{\prime}}\right)$ are mostly reported for wall-resolved LES, whereas most wall-modelled LES focus on the mean quantities, i.e. velocity profiles $(\bar{u})$, lift coefficient $\left(C_{L}\right)$, drag coefficient $\left(C_{D}\right)$ and pressure coefficient $\left(C_{p}\right)$. Notable exceptions are Kawai \& Asada (2013) which reports $C_{f}$ and $\overline{u^{\prime} v^{\prime}}$ in WMLES of flow past the A-airfoil, and George \& Lele (2014) which reports on diagonal components of the Reynolds stress tensor in WMLES of flow past a NACA0012 airfoil. One may discern from these studies that accurate predictions of the skin-friction coefficient and Reynolds stresses are somewhat challenging. Also listed in table 1 is the spanwise domain extent $L_{z} / C$. It was noted in DNS of flow past an airfoil (Zhang \& Samtaney 2016), that the spanwise domain size has a significant impact on the results.

\section{Wall modelling in complex geometry}

In this section, starting with the Navier-Stokes equations in the generalized curvilinear coordinates, we apply near-wall filtering along with the assumption of 
inner scaling to derive an ordinary differential equation (ODE) governing the local wall-normal velocity gradient, and a slip Dirichlet boundary condition for velocity at a virtual wall.

\subsection{Navier-Stokes equations}

The incompressible Navier-Stokes $(\mathrm{N}-\mathrm{S})$ equations in the generalized curvilinear coordinates are

$$
\begin{gathered}
\frac{\partial U^{m}}{\partial \xi^{m}}=0, \\
\frac{\partial\left(\sqrt{g} u_{i}\right)}{\partial t}+\frac{\partial F_{i}^{m}}{\partial \xi^{m}}=0,
\end{gathered}
$$

where $U^{m}$ (the volume flux normal to the surface of constant $\xi^{m}$ ) and $F_{i}^{m}$ are given by

$$
\left.\begin{array}{c}
U^{m}=\sqrt{g} \frac{\partial \xi^{m}}{\partial x_{j}} u_{j}, \\
F_{i}^{m}=U^{m} u_{i}+\sqrt{g} \frac{\partial \xi^{m}}{\partial x_{i}} p-v G^{m n} \frac{\partial u_{i}}{\partial \xi^{n}}, \\
\sqrt{g}=J^{-1}=\operatorname{det}\left[\frac{\partial x_{i}}{\partial \xi^{j}}\right], \quad G^{m n}=\sqrt{g} \frac{\partial \xi^{m}}{\partial x_{r}} \frac{\partial \xi^{n}}{\partial x_{r}},
\end{array}\right\}
$$

where $p$ is the pressure, $u_{i}$ is the velocity in the Cartesian coordinates, $v$ is the kinematic viscosity, $J^{-1}$ is the Jacobian of the transformation, $G^{m n}$ is the mesh skewness tensor.

Applying a nominal filter to the $\mathrm{N}-\mathrm{S}$ equations, the filtered LES equations have a form similar to (3.1) and (3.2), and are written below in terms of the resolved velocity field,

$$
\begin{gathered}
\tilde{U}^{m}=\sqrt{g} \frac{\partial \xi^{m}}{\partial x_{j}} \tilde{u}_{j}, \\
\tilde{F}_{i}^{m}=\tilde{U}^{m} \tilde{u}_{i}+\sqrt{g} \frac{\partial \xi^{m}}{\partial x_{i}} \tilde{p}-v G^{m n} \frac{\partial \tilde{u}_{i}}{\partial \xi^{n}}+\sqrt{g} \frac{\partial \xi^{m}}{\partial x_{j}} T_{i j},
\end{gathered}
$$

where $\sim$ denote filtered quantities, $T_{i j}=\widetilde{u_{i} u_{j}}-\tilde{u}_{i} \tilde{u}_{j}$ is the SGS stress tensor.

\subsection{Near-wall filtering}

Following Chung \& Pullin (2009), we define two near-wall filters in the near-wall region,

$$
\begin{gathered}
\tilde{\phi}(\xi, \eta, \zeta, t)=\iint \phi(\xi, \eta, \zeta, t) G\left(\xi-\xi^{\prime}, \Delta_{f}\right) G\left(\zeta-\zeta^{\prime}, \Delta_{f}\right) \mathrm{d} \xi^{\prime} \mathrm{d} \zeta^{\prime}, \\
\langle\phi\rangle=\frac{1}{h} \int_{0}^{h} \tilde{\phi}(\xi, \eta, \zeta, t) \mathrm{d} \eta,
\end{gathered}
$$

where (3.6) and (3.7) define the wall-parallel filter and the wall-normal averaging filter, respectively. The planar filtering (with filtering function $G$ ) is purely formal; 
we do not perform such a filtering operation and indeed no explicit filtering of the velocity or pressure fields is employed in the present approach. It should be noted that, consistent with other approaches involving body-fitted mesh computations, the computational mesh is constrained to be locally orthogonal to the airfoil surface for wall-normal averaging, and the wall-normal distance is denoted as $y_{n}$. As shown in figure 2, the distance, $h$ above is typically chosen as the distance of the first grid point from the airfoil surface or the solid wall; and $h_{0}$ is the height of the virtual wall that is further discussed below. Applying the wall-parallel filter (3.6) to the momentum equations (3.2), we obtain

$$
\begin{aligned}
& \frac{\partial \tilde{u}}{\partial t}=-\frac{1}{\sqrt{g}} \frac{\partial}{\partial \xi^{m}}\left(\widetilde{U^{m} u}+\sqrt{g} \frac{\partial \xi^{m}}{\partial x} \tilde{p}-v G^{m n} \frac{\partial \tilde{u}}{\partial \xi^{n}}\right), \\
& \frac{\partial \tilde{v}}{\partial t}=-\frac{1}{\sqrt{g}} \frac{\partial}{\partial \xi^{m}}\left(\widetilde{U^{m} v}+\sqrt{g} \frac{\partial \xi^{m}}{\partial y} \tilde{p}-v G^{m n} \frac{\partial \tilde{v}}{\partial \xi^{n}}\right), \\
& \frac{\partial \tilde{w}}{\partial t}=-\frac{1}{\sqrt{g}} \frac{\partial}{\partial \xi^{m}}\left(\widetilde{U^{m} w}+\sqrt{g} \frac{\partial \xi^{m}}{\partial z} \tilde{p}-v G^{m n} \frac{\partial \tilde{w}}{\partial \xi^{n}}\right),
\end{aligned}
$$

where $(u, v, w)=\left(u_{1}, u_{2}, u_{3}\right)$ denote the velocity components in the Cartesian coordinates.

\subsection{Inner scaling assumption and governing equation for $\eta_{0}$}

The virtual wall model formulation is essentially based on the inner scaling ansatz, which states that the near-wall turbulent statistics are characterized by the kinematic viscosity $v$ and the local friction velocity $u_{\tau}(x, y, z, t)$. For attached boundary layers, the inner scaling ansatz works well until the end of the log-law region $(15 \%$ of boundary layer thickness and somewhat less for APG boundary layers), which is certainly sufficient for WMLES. For separated flows, a modified wall model, still essentially based on the inner scaling ansatz, was successfully tested by Cheng et al. (2015) for a flat-plate turbulent boundary layer with separation/reattachment.

Presently, we define the magnitude of the resultant velocity $\tilde{q}$ and velocity angle $\theta$ on the wall-parallel plane as

$$
\tilde{q}=\sqrt{\tilde{u}_{s}^{2}+\tilde{w}^{2}}, \quad \theta=\arccos \left(\tilde{u}_{s} / \tilde{q}\right)
$$

where $\tilde{u}_{s}$ is the streamwise velocity along the airfoil surface (as shown in figure 2),

$$
\tilde{u}_{s}=\tilde{u} \cos \theta_{w}+\tilde{v} \sin \theta_{w},
$$

where $\theta_{w}$ denotes the local angle between the airfoil surface and the $x$-coordinate. We assume $\tilde{q}$ follows inner scaling, i.e.

$$
\tilde{q}(\xi, \eta, \zeta, t)=u_{\tau}(\xi, \zeta, t) F\left(y_{n}^{+}\right), \quad y_{n}^{+}=u_{\tau} y_{n} / v
$$

where $F\left(y_{n}^{+}\right)$is an unknown function of the normal distance from the airfoil in wall units, $u_{\tau}=\sqrt{\nu \eta_{0}}$ is the friction velocity and $\eta_{0}(\xi, \zeta, t)$ is the wall-normal gradient of $\tilde{q}$ defined as

$$
\left.\eta_{0} \equiv \frac{\partial \tilde{q}}{\partial y_{n}}\right|_{w}
$$




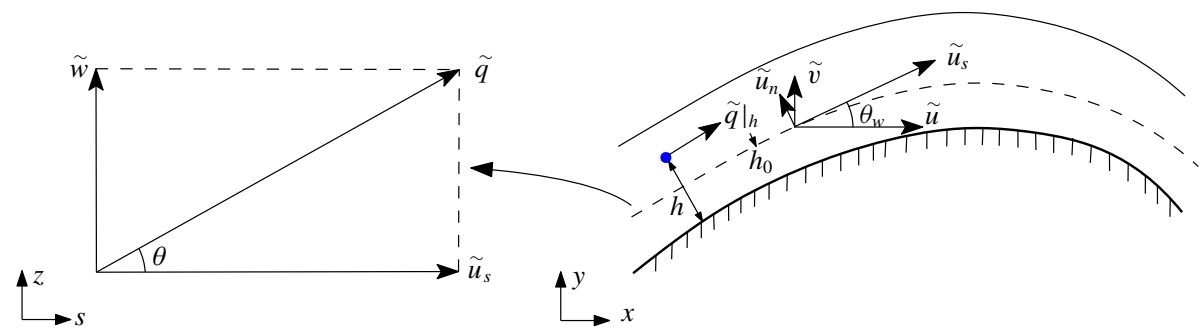

FIGURE 2. (Colour online) Sketch of the near-wall velocity components. The dashed line upon the solid wall denotes the virtual wall, and the blue point denotes the centre of the first grid cell off the solid wall.

The following ODEs can then be derived as

$$
\frac{\partial u_{\tau}}{\partial \eta_{0}}=\frac{v}{2 u_{\tau}}=\frac{1}{2} \sqrt{\frac{v}{\eta_{0}}}, \quad \frac{\partial y_{n}^{+}}{\partial \eta_{0}}=\frac{y_{n}^{+}}{2 \eta_{0}} .
$$

Applying the wall-normal averaging filter (3.7) to the governing equation of $\tilde{q}$ results in

$$
\frac{\partial\langle q\rangle}{\partial t}=\frac{\left.\tilde{q}\right|_{h}}{2 \eta_{0}} \frac{\partial \eta_{0}}{\partial t},
$$

where $\left.\tilde{q}\right|_{h}=u_{\tau} F\left(h^{+}\right)$is the resolved resultant velocity at a distance $h$ from the solid wall (see figure 2). It should be noted that (3.14) is an exact consequence of (3.7) and (3.11). Moreover, an explicit form of $F\left(y_{n}^{+}\right)$is not required owing to cancellation.

From the definition of $\tilde{q}$, (3.9), we have

$$
\frac{\partial\langle q\rangle}{\partial t}=\frac{1}{h} \int_{0}^{h}\left(\frac{\tilde{u}_{s}}{\tilde{q}} \frac{\partial \tilde{u}_{s}}{\partial t}+\frac{\tilde{w}}{\tilde{q}} \frac{\partial \tilde{w}}{\partial t}\right) \mathrm{d} y_{n} .
$$

Some terms in the above equation may be analytically integrated (see details in appendix A). Then combining (3.14) and (3.15), we arrive at the governing equation for $\eta_{0}$,

$$
\frac{\partial \eta_{0}}{\partial t}=C_{1} \eta_{0}-C_{2} \eta_{0}^{2}
$$

where

$$
C_{1}=\frac{2}{\left.\tilde{q}\right|_{h}}\left(F_{\xi}+F \zeta+\left.M \frac{\nu G^{22}}{\sqrt{g} h} \frac{\partial \tilde{q}}{\partial y_{n}}\right|_{h}\right), \quad C_{2}=\frac{2 \nu G^{22}}{\left.\sqrt{g} h \tilde{q}\right|_{h}}
$$

Detailed expressions for $F_{\xi}, F_{\zeta}$ and $M$ are given by equations (A 3) and (A 5) and (A 7), respectively, in appendix A.

\subsection{Near-wall treatment and solution of the $O D E$}

On the right-hand side of (3.17), $F_{\xi}, F_{\zeta}$ and $M$ are unknown, both of which are estimated by resolved-scale quantities at the first grid point $\left(y_{n}=h\right)$ above the wall. For example, the first term on the right-hand side of (A 3) is approximated by LES 
resolved-scale values at $y_{n}=h$ as

$$
\begin{aligned}
- & \frac{1}{h} \int_{0}^{h} \frac{\tilde{u}_{s}}{\tilde{q}} \frac{\cos \theta_{w}}{\sqrt{g}} \frac{\partial}{\partial \xi^{1}}\left(\widetilde{U^{1} u}+\sqrt{g} \frac{\partial \xi^{1}}{\partial x} \tilde{p}-v G^{11} \frac{\partial \tilde{u}}{\partial \xi^{1}}\right) \mathrm{d} y_{h} \\
& \approx-\left.\frac{\tilde{u}_{s}}{\tilde{q}} \frac{\cos \theta_{w}}{\sqrt{g}}\right|_{h} \frac{\partial}{\partial \xi^{1}}\left[\left.\widetilde{U^{1} u}\right|_{h}+\left.\left(\sqrt{g} \frac{\partial \xi^{1}}{\partial x} \tilde{p}\right)\right|_{h}-\left.\left(v G^{11} \frac{\partial \tilde{u}}{\partial \xi^{1}}\right)\right|_{h}\right],
\end{aligned}
$$

where

$$
\left.\begin{array}{c}
\left.\widetilde{U^{1} u}\right|_{h}=\left.\sqrt{g} \frac{\partial \xi^{1}}{\partial x_{1}} \widetilde{u u}\right|_{h}+\left.\sqrt{g} \frac{\partial \xi^{1}}{\partial x_{2}} \widetilde{u v}\right|_{h}+\left.\sqrt{g} \frac{\partial \xi^{1}}{\partial x_{3}} \widetilde{u w}\right|_{h}, \\
\left.\left.\right|_{h} \approx \tilde{u} \tilde{u}\right|_{h}+\left.T_{x x}\right|_{h},\left.\left.\widetilde{u v}\right|_{h} \approx \tilde{u} \tilde{v}\right|_{h}+\left.T_{x y}\right|_{h},\left.\left.\widetilde{u w}\right|_{h} \approx \tilde{u} \tilde{w}\right|_{h}+\left.T_{x z}\right|_{h},
\end{array}\right\}
$$

where $T_{x x}, T_{x y}$ and $T_{x z}$ are the SGS stress tensor components (discussed in $\S 4.1$ ). The other terms in $F_{\xi}$ (A 3) and $F_{\zeta}$ (A 5) and $M$ (A 7) are approximated in a similar manner.

If coefficients $C_{1}$ and $C_{2}$ in equation (3.16) are weakly dependent on $t$, then assuming these to be constant, equation (3.16) may be interpreted as a second-order linear ODE for $\eta_{0}$ which can be solved analytically (details are in appendix B).

Once $\eta_{0}(\xi, \zeta, t)$ is known, and the velocity angle $\theta(\xi, \zeta, t)$ is estimated as $\arccos \left(\left.\tilde{u}_{s}\right|_{h} /\left.\tilde{q}\right|_{h}\right.$ ) (meaning that streamline orientation on the virtual wall is determined by the first grid cell from the resolved LES field, an approximation justified below based on the work of Cheng et al. (2015)), the local wall shear stress components may then be computed as

$$
\tau_{w, s}=\mu \eta_{0} \cos \theta, \quad \tau_{w, z}=\mu \eta_{0} \sin \theta .
$$

Here $\mu=\rho v$ is the dynamic viscosity, and $\boldsymbol{\tau}_{w} \equiv\left(\tau_{w, s}, \tau_{w, z}\right)$ is the LES representation of the surface stress vector. Above, we make the approximation that the velocity angle $\theta$ is assumed to be constant within the first grid cell, $0 \leqslant y_{n} \leqslant h$. Cheng et al. (2015) proposed an algebraic model for $\theta$ in turbulent boundary layer simulations and concluded that there is little difference between the constant velocity angle model and the algebraic model. In the present paper, the constant velocity angle model is adopted for simplicity.

\subsection{Slip velocity boundary conditions}

Once $\eta_{0}$ is solved using (3.16), we complete the wall model with a slip velocity at a raised virtual wall plane located at $\eta=h_{0}$, which scales with the boundary layer thickness but remains small, i.e. $h_{0} \leqslant 0.1 \delta$. Typically, $h_{0}$ is chosen as a small fraction of the near-wall cell size. In previous studies, both in channel flow by Chung \& Pullin (2009) and turbulent boundary layer flows (ZPG and APG) by Inoue \& Pullin (2011) and Cheng et al. (2015), $h_{0} / \Delta \eta=0.18$ is fixed with respect to the first off-wall grid cell and the sensitivity to changes was documented in Chung \& Pullin (2009). Their verifications and validations capture the near-wall flow physics well, and we follow this choice in the present research.

We model the resultant slip velocity $\left.\tilde{q}\right|_{h_{0}}$ on the virtual wall as

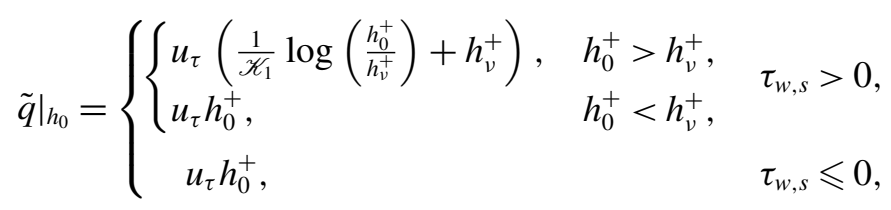


where $h_{0}^{+}=u_{\tau} h_{0} / v, h_{v}^{+}$is the intercept between the linear and log components in the law of the wall. Experimental research shows that the outer edge of the viscous sublayer is located at $h_{v}^{+} \approx 11$, which is approximately equivalent to the offset $(=5.0)$ in the classical $\log$ law. This value was adopted by Chung \& Pullin (2009) and Inoue \& Pullin (2011), and also by Cheng et al. (2015) in modelling the turbulent boundary layer flows with separation and reattachment. Presently, $h_{v}^{+}=11$ is used as an empirical parameter in the wall model. In the attached region $\left(\tau_{w, s}>0\right)$, the linear-log relation is essentially the same as Chung \& Pullin (2009), which is derived from stretched-vortex SGS model (discussed below) based on the detached/attached eddy concepts of Townsend (1976). The Kármán-like constant $\mathscr{K}_{1}$ is dynamically computed (refer to Chung \& Pullin (2009) for more details). In the separated region, $\left(\tau_{w, s} \leqslant 0\right)$, the log-like relation is no longer valid and Cheng et al. (2015) proposed a linear relationship which appears to work reasonably well in regions of flow separation. Presently, we follow the linear law by Cheng et al. (2015).

\subsection{Summary of the wall model}

The wall model is summarized as follows: in the near-wall region, equation (3.16) is solved for $\eta_{0}$, in which the coefficients on the right-hand side are approximated with the resolved LES field at the first grid cell, i.e. $h=h_{0}+\Delta y_{n} / 2$, equation (3.21) is then used to compute the resultant velocity $\left.\tilde{q}\right|_{h_{0}}$ on the virtual wall with the streamwise and spanwise velocity components given by

$$
\left.\tilde{u}_{s}\right|_{h_{0}}=\left.\tilde{q}\right|_{h_{0}} \cos \theta,\left.\quad \tilde{w}\right|_{h_{0}}=\left.\tilde{q}\right|_{h_{0}} \sin \theta .
$$

The contribution of the wall-normal velocity component $\left.\tilde{u}_{n}\right|_{h_{0}}$ to $\tilde{u}$ and $\tilde{v}$ is assumed to be small compared with $\left.\tilde{u}_{s}\right|_{h_{0}}$, and presently we use $\left.\tilde{u}_{n}\right|_{h_{0}}=0$. Finally, the slip velocity boundary condition on the virtual wall $\eta=h_{0}$ is

$$
\left.\tilde{u}\right|_{h_{0}}=\left.\tilde{q}\right|_{h_{0}} \cos \theta \cos \theta_{w},\left.\quad \tilde{v}\right|_{h_{0}}=\left.\tilde{q}\right|_{h_{0}} \cos \theta \sin \theta_{w},
$$

with the spanwise velocity component $\left.\tilde{w}\right|_{h_{0}}$ given by (3.22).

\section{SGS model and numerical set-up}

\subsection{Stretched-vortex SGS model}

The stretched-vortex SGS model has been previously widely deployed in LES of wallbounded turbulent flows by Pullin and co-workers. Here, for the sake of completeness, we present the essential features of this structure-based SGS model, which assumes that the turbulent fine scales are composed of tube-like structures with spiral vortices (Lundgren 1982). In each computational cell, the ensemble dynamics is dominated by a stretched vortex with orientation $\boldsymbol{e}^{\nu}$, taken from a delta-function probability density function (Misra \& Pullin 1997). Consequently, the SGS stress tensor $T_{i j}$ is modelled as (Chung \& Pullin 2009)

$$
T_{i j}=\left(\delta_{i j}-e_{i}^{v} e_{j}^{v}\right) K, \quad K=\int_{k_{c}}^{\infty} E(k) \mathrm{d} k,
$$

where $K$ is the SGS kinetic energy, $k_{c}=\pi / \Delta_{c}$ is the cutoff wavenumber $\left(\Delta_{c}=\right.$ $\left(\Delta_{x} \Delta_{y} \Delta_{z}\right)^{1 / 3}$ ) and $E(k)$ is the SGS energy spectrum given by Lundgren (1982). The integration of the energy spectrum gives

$$
K=\frac{1}{2} \mathcal{K}_{0}^{\prime} \Gamma\left[-1 / 3, \kappa_{c}^{2}\right], \quad \mathcal{K}_{0}^{\prime}=\mathcal{K}_{0} \epsilon^{2 / 3} \lambda_{v}^{2 / 3}, \quad \lambda_{v}=(2 v / 3|\tilde{a}|)^{1 / 2}, \quad \kappa_{c}=k_{c} \lambda_{v}, \quad(4.2 a-d)
$$


where $\Gamma$ is the incomplete gamma function, $\tilde{a}=e_{i}^{v} e_{j}^{v} \tilde{S}_{i j}$ is the stretching felt along the subgrid vortex axis imposed by the resolved scales and $\tilde{S}_{i j}$ is the resolved SGS strain tensor. The composite parameter $\mathcal{K}_{0}^{\prime}$ is obtained dynamically by structure-function matching at the grid-scale cutoff (Voelkl, Pullin \& Chan 2000), i.e. $\mathcal{K}_{0}^{\prime}=\left\langle F_{2}\right\rangle /\left\langle Q\left(\kappa_{c}, d\right)\right\rangle$, where $\langle$.$\rangle denotes a local-averaging operator computed$ from the neighbouring 26 points, $F_{2}$ is the second-order velocity structure function from the resolved LES field, and the calculation of $Q\left(\kappa_{c}, d\right)$ is similar to Voelkl et al. (2000), Chung \& Pullin (2009) with $\kappa_{c}=r / \Delta_{c}$ and $r$ is the distance from the neighbouring point to the vortex axis.

The stretched-vortex SGS model coupled with the virtual wall model have been together applied to several canonical turbulent flows relevant to the present flow: Chung \& Pullin (2009) for turbulent channel flow; Inoue \& Pullin (2011) for turbulent boundary layer flow at arbitrarily large $R e$; Inoue et al. (2012) for combining a predictive-wall model with LES; Inoue et al. (2013) for adverse-pressure turbulent boundary layers; Saito et al. (2012), Saito \& Pullin (2014) and Sridhar, Pullin \& Cheng (2017) for rough-wall turbulent boundary layers; Cheng et al. (2015) for separated-reattached turbulent boundary layers. However, all the previous works based on this approach are applied to simple canonical geometries. The extension and testing of the models to complex geometries are necessary to pave the way to more engineering applications.

\subsection{Numerical method}

The governing equations (3.4) and (3.5) are discretized as

$$
\left.\begin{array}{l}
\frac{\delta \tilde{U}^{m}}{\delta \xi^{m}}=0, \\
-\frac{1}{2}\left(C_{i}^{n-1}+S_{i}^{n-1}\right)+R_{i}\left(\tilde{p}^{n+1}\right)+D_{i}\left(\tilde{u}^{n+1}\right),
\end{array}\right\}
$$

where $\delta / \delta \xi^{m}$ represents the energy conservative fourth-order finite difference operator (Morinishi et al. 1998), $C_{i}$ and $S_{i}$ represent the convective term and SGS term, $D_{i}$ and $R_{i}$ are discrete operators for the viscous term and the pressure gradient term, respectively. These quantities are

$$
\left.\begin{array}{c}
C_{i}=-\frac{\delta}{\delta \xi^{m}}\left(\tilde{U}^{m} \tilde{u}_{i}\right), \quad S_{i}=-\frac{\delta}{\delta \xi^{m}}\left(\sqrt{g} \frac{\delta \xi^{m}}{\delta x_{j}} T_{i j}\right), \\
R_{i}=-\frac{\delta}{\delta \xi^{m}}\left(\sqrt{g} \frac{\delta \xi^{m}}{\delta x_{i}}\right), \quad D_{i}=\frac{\delta}{\delta \xi^{m}}\left(\nu G^{m n} \frac{\delta}{\delta \xi^{n}}\right),
\end{array}\right\}
$$

where the convective term is computed in the skew-symmetric form to minimize the aliasing error (Zang 1991; Morinishi et al. 1998). The fractional step method (Zang, Street \& Koseff 1994; Zhang et al. 2015) is used to solve the governing equations. This method follows the predictor-corrector procedure, and the pressure Poisson equation is solved using the multigrid method with line-relaxed Gauss-Seidel as a smoother. The code is parallelized using standard MPI-protocol. To achieve near-optimal load balancing, the mesh is divided into blocks of equal size and each of them is assigned to a unique processor. All the simulations are performed on the Shaheen-Cray XC40 at KAUST. The DNS code (without the SGS stress terms) with the same method is described in Zhang et al. (2015) for flow past an airfoil, and is the one used for verification in the low $R e_{c}$ case in the present paper. The WRLES code with the same method was previously applied successfully in flow past smooth and grooved cylinders (Cheng et al. 2017, 2018a). 


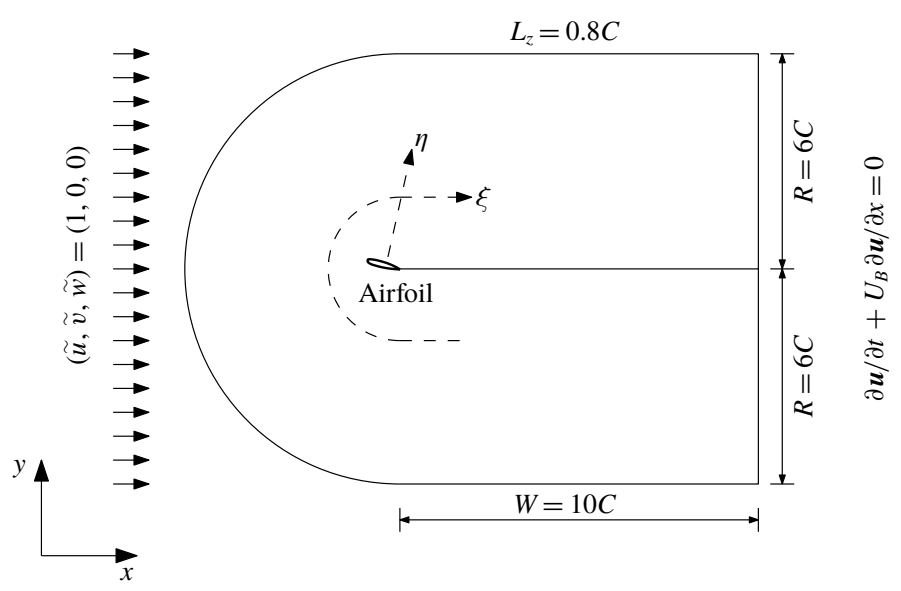

FIGURE 3. Sketch of the numerical set-up and computational domain.

\begin{tabular}{lccccccr}
\hline Airfoil & Method & $R e_{c}$ & AoA (deg.) & $N_{\xi} \times N_{\eta} \times N_{z}$ & $\Delta \xi_{\max }^{+}$ & $\Delta \eta_{\max }^{+}$ & $\Delta z_{\max }^{+}$ \\
NACA0012 & DNS & $10^{4}$ & 5 & $2048 \times 256 \times 256$ & 7.4 & 0.8 & 8.8 \\
NACA0012 & WMLES & $10^{4}$ & 5 & $768 \times 96 \times 64$ & 19.7 & 14.2 & 38.4 \\
NACA0018 & WMLES & $10^{5}$ & 5 & $1600 \times 128 \times 128$ & 54.9 & 15.8 & 65.8 \\
A-Airfoil & WMLES & $2.1 \times 10^{6}$ & 13.3 & $3200 \times 256 \times 256$ & 80.1 & 16.4 & 85.4
\end{tabular}

TABLE 2. Summary of the performed numerical cases.

\subsection{Numerical set-up}

The numerical set-up and domain size are illustrated in figure 3. It should be noted that a sufficiently long spanwise domain size $\left(L_{z}\right)$ is important for the proper development of 3-D turbulent structures, and the associated turbulent statistics. Kitsios et al. (2011) performed LES of flow past the NACA0015 airfoil at $R e_{c}=3 \times 10^{4}$ with different $L_{z}$ and found that $L_{z}=0.66 C$ was adequate. It was concluded that the impact of large-scale structures reduces as $R e_{c}$ increases. Zhang \& Samtaney (2016) presented a comprehensive assessment of domain size effects in DNS of flow past the NACA0012 airfoil at $R e_{c}=5 \times 10^{4}$, and recommended a spanwise size of $L_{z}=0.8 C$. It is found that a smaller $L_{z}$ tends to under-predict the turbulent fluctuations near the separation point but over-predict them inside the separation bubble. Presently, $L_{z}=0.8 \mathrm{C}$ is adopted in all the simulations to enforce the correct spanwise periodicity and capture large-scale turbulent structures. It is by far the largest spanwise extent in LES of airfoil flows at high $\operatorname{Re}_{c}$ (see table 1).

For boundary conditions, a uniform flow $(\tilde{u}, \tilde{v}, \tilde{w})=\left(U_{\infty}, 0,0\right), U_{\infty}=1$ is imposed at the inlet, and the convective boundary condition $\partial \boldsymbol{u} / \partial t+U_{B} \partial \boldsymbol{u} / \partial x=0$ is used at the outflow plane, where $U_{B}$ is the bulk velocity. The slip velocity from the wall model is specified at the virtual wall, and the periodic boundary condition is assumed in the spanwise direction.

Three cases as summarized in table 2 are performed to verify and validate the wall model. The low $R e_{c}$ case (NACA0012, $R e_{c}=10^{4}, A o A=5^{\circ}$ ) is performed with both DNS and WMLES, and the numerical results from DNS are utilized to verify the performance of the wall model at low $R e_{c}$. To check the effects of mesh resolution on the WMLES, a mesh convergence study of this case with WMLES 

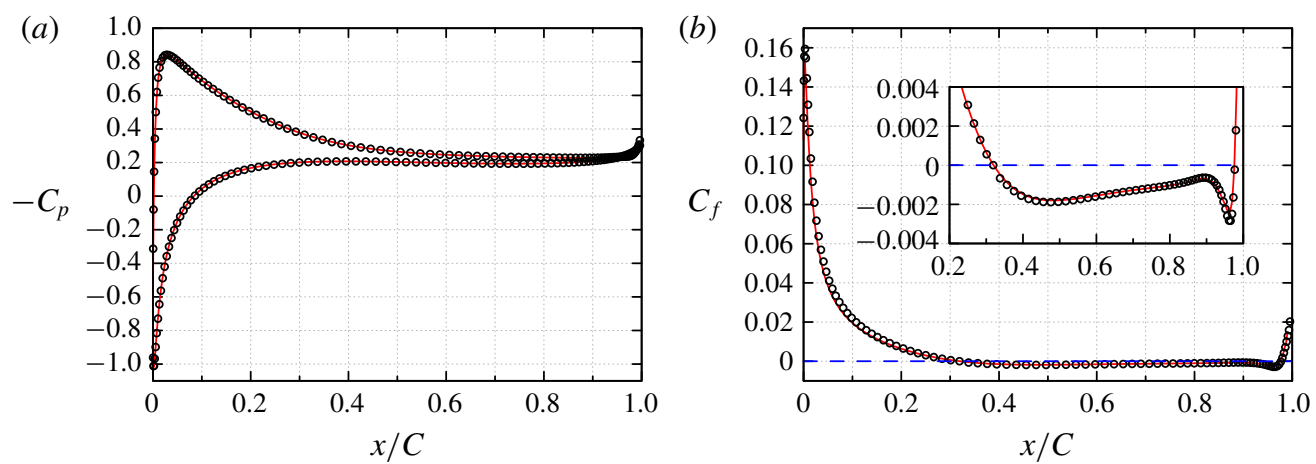

FIgURE 4. (Colour online) NACA0012, $R e_{c}=10^{4}$. (a) Distribution of the pressure coefficient $C_{p}$ around the airfoil, and $(b)$ skin-friction coefficient $C_{f}$ on the suction surface. — zero crossing of this line indicates separation and reattachment.

is presented in appendix C. For the higher Reynolds number cases, DNS becomes computationally prohibitive, and for these cases we compare results from WMLES with experiments with an emphasis on strong validation. This somewhat limits our choice of experiments to those which have presented skin-friction coefficient and Reynolds stress components. The relatively moderate $R e_{c}$ case (NACA0018, $R e_{c}=10^{5}$, $A o A=5^{\circ}$ ) is validated with the experimental results from Boutilier \& Yarusevych (2012) and Kirk \& Yarusevych (2017). The Aérospatiale A-airfoil near stall condition $\left(R e_{c}=2.1 \times 10^{6}, A o A=13.3^{\circ}\right)$ is a benchmark case from the Brite-Euram project LESFOIL (Mellen, Frögrave \& Rodi 2003; Mary \& Sagaut 2002). This somewhat challenging case has been extensively used to assess RANS and LES models (Schmidt \& Thiele 2003; Chaouat 2006; Kawai \& Asada 2013), and is also for validation in the present paper. In the experiment of the A-airfoil, the boundary layer of the pressure surface is tripped at around $x / C=0.3$ (Dahlstrom \& Davidson 2001). These trips, whose height is smaller than the first wall-adjacent grid spacing, are not included in the our simulations. The numerical noise would act as the perturbation source, and allow natural transition to turbulence. This choice is same as Park \& Moin (2014) in WMLES of the NACA4412 airfoil at $R e_{c}=1.64 \times 10^{6}$, in which reasonable prediction of transition onset was reported without special treatment of the boundary layer trips.

\section{Verification and validation}

In this section, we provide verification of one case $\left(R e_{c}=10^{4}\right)$ with DNS, and then strong validation of the larger $R e_{c}$ cases against experimental results.

\subsection{Comparison between DNS and WMLES: $R e_{c}=10^{4}$}

The time- and spanwise-averaged pressure coefficient $\left(C_{p}\right)$ and skin-friction coefficient $\left(C_{f}\right)$ are computed as

$$
C_{p}=\frac{\bar{p}-p_{\infty}}{\frac{1}{2} \rho U_{\infty}^{2}}, \quad C_{f}=\frac{\bar{\tau}_{w, s}}{\frac{1}{2} \rho U_{\infty}^{2}},
$$

where $\bar{p}$ and $\bar{\tau}_{w, s}$ are the time- and spanwise-averaged wall pressure and streamwise wall shear stress, $p_{\infty}$ is the reference pressure taken at the outlet boundary. The $C_{p}$ and $C_{f}$ for this NACA0012 case are shown in figure 4. The present WMLES results 


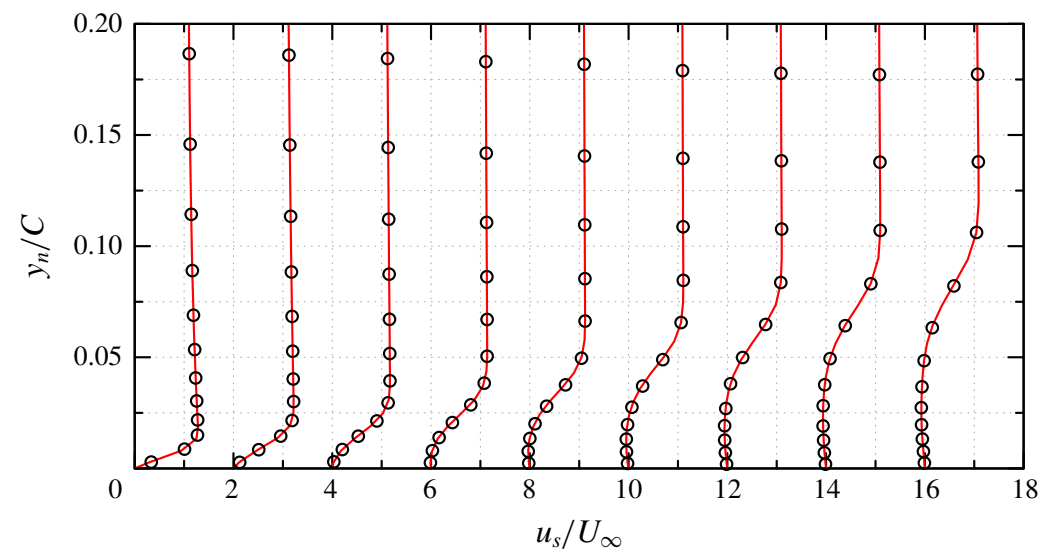

FIGURE 5. (Colour online) NACA0012, $R e_{c}=10^{4}$. The mean streamwise velocity profiles along the wall-normal lines at nine locations. From left to right, $x / C=0.1-0.9$ with equal distances of 0.1 . The plots are shifted by $0,2, \ldots, 16$ for clarity. — , present DNS; $\bigcirc$, present WMLES.
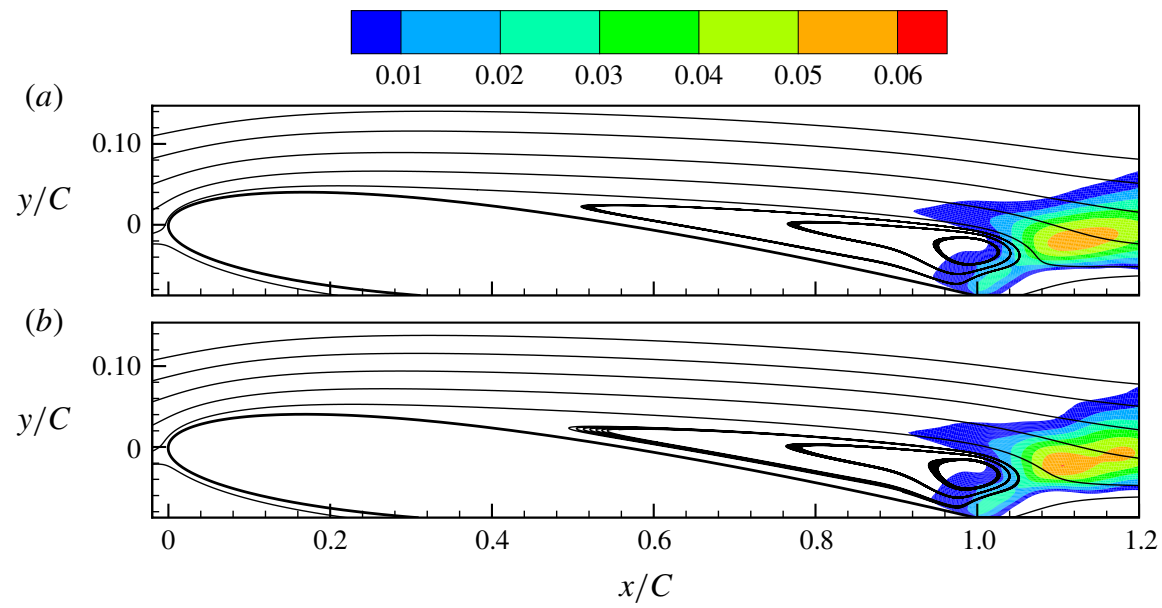

FIgURE 6. (Colour online) NACA0012, $R e_{c}=10^{4}$. Distribution of the time- and spanwise-averaged Reynolds stress $-\overline{u^{\prime} v^{\prime}} / U_{\infty}^{2}=[0.001,0.06]$ : (a) DNS result, and (b) present WMLES result. The transition onset is indicated by the threshold value 0.001 .

compare well with the DNS results with negligible difference. The skin-friction coefficient profile indicates separation and reattachment at $x / C=0.32$ and $x / C=0.98$, respectively. The streamwise mean velocity profiles at nine locations along the suction surface $(x / C=0.1-0.9$ with equal distances of 0.1$)$ are shown in figure 5 . The present WMLES results indicate that its prediction of both separation and reattachment are in good agreement with that of the DNS predictions.

Colour contours of time- and spanwise-averaged off-diagonal Reynolds stress $-\overline{u^{\prime} v^{\prime}} / U_{0}^{2}$ within the range $[0.001,0.06]$ superimposed on average streamline contours are shown in figure 6. Here the threshold value of 0.001 is chosen as an indicator for transition onset, which is commonly used in various numerical (Zhou \& Wang 2012) 

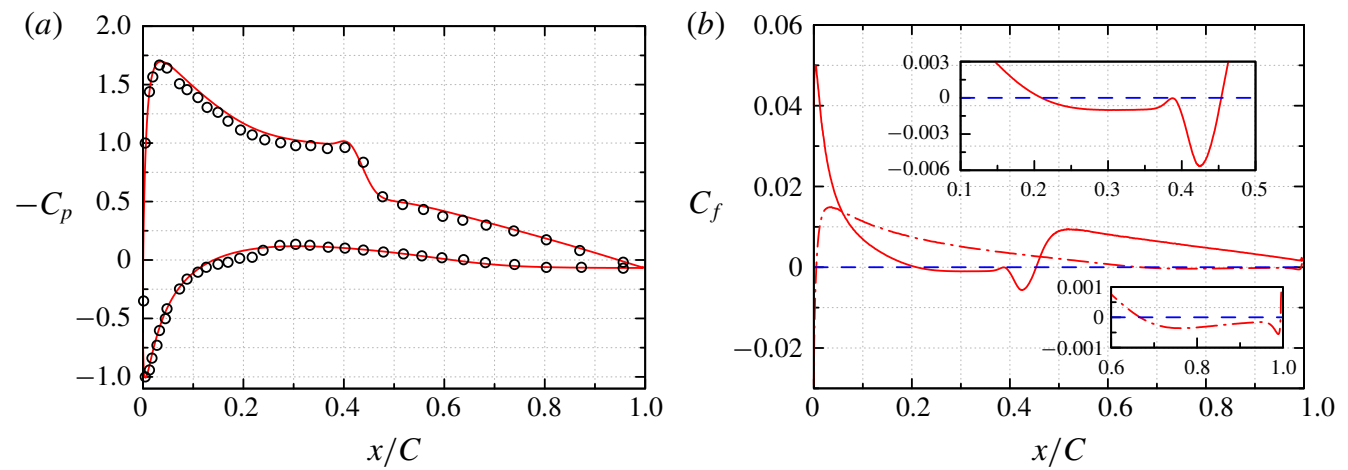

FIgURE 7. (Colour online) NACA0018, $R e_{c}=10^{5}$. (a) Distribution of the pressure coefficient $C_{p}$, and $(b)$ skin-friction coefficient $C_{f}$ around the airfoil. $\bigcirc$, experimental data from Kirk \& Yarusevych (2017); — , corresponding results from the present WMLES; - - -,$C_{f}$ on the pressure side from the present WMLES. The - - - line $C_{f}=0$ is shown for convenience: zero crossing of this line indicates separation and reattachment. The experimental values of the separation and reattachment point on the suction side are estimated to be located at $x / C=0.24 \pm 0.02,0.52 \pm 0.02$, respectively (Kirk \& Yarusevych 2017).

and experimental investigations (Buchmannand, Atkinson \& Soria 2013) of flow past bluff bodies. The transition onset occurs near the trailing edge, i.e. $x / C=0.92$ and $x / C=0.91$ for DNS and WMLES, respectively.

\subsection{Validation of WMLES: $R e_{c}=10^{5}$}

The pressure coefficient $C_{p}$ and skin-friction coefficient $C_{f}$ for the NACA0018 case are shown in figure 7 . The WMLES $C_{p}$ results compare well with the experiment. The separation and reattachment on the pressure side occur at $x / C=0.67,0.99$, and $x / C=0.21,0.45$ on the suction side. In the experiment by Kirk \& Yarusevych (2017), the separation and reattachment points on the suction side are estimated to be located at $x / C=0.24 \pm 0.02,0.52 \pm 0.02$ : these are inferred indirectly from the pressure distributions - a flat distribution is expected within the recirculating region. The trailing edge separation on the pressure side is not investigated in the experimental research, however, our WMLES predicts a trailing edge separation on the pressure side, in qualitative agreement with another experiment by Nakano et al. (2006) with the same airfoil geometry but slightly different $\operatorname{Re}_{c}\left(=1.6 \times 10^{5}\right)$ and $A o A\left(=6^{\circ}\right)$.

The mean velocity profiles in the $x$-direction at different locations along the suction surface $(x / \bar{C}=0.2-0.48$ with equal distances of 0.02$)$ are shown in figure $8(a)$, here $\bar{C}$ is the $x$-axis projection of the chord length. The mean velocity profiles in the attached zone, i.e. $x / C=0.50,0.52,0.54,0.56,0.60,0.66,0.73,0.87$ are shown in figure $8(b)$. Good agreement between our WMLES results and the experiment is noted.

The Reynolds stress component, $\sqrt{\overline{u^{\prime} u^{\prime}}} / U_{\infty}$, on the suction side is shown in figure 9, at the same locations as the mean velocity profiles. The LES results match quite well with the experimental data in the range of $[0.2,0.38]$ and $[0.5,0.87]$. We note difference between LES and experiments in the range of $[0.38,0.48]$, close to the reattachment point. It should be emphasized that both the present LES and experiments in this range show a sharp drop in pressure coefficient distributions (see 

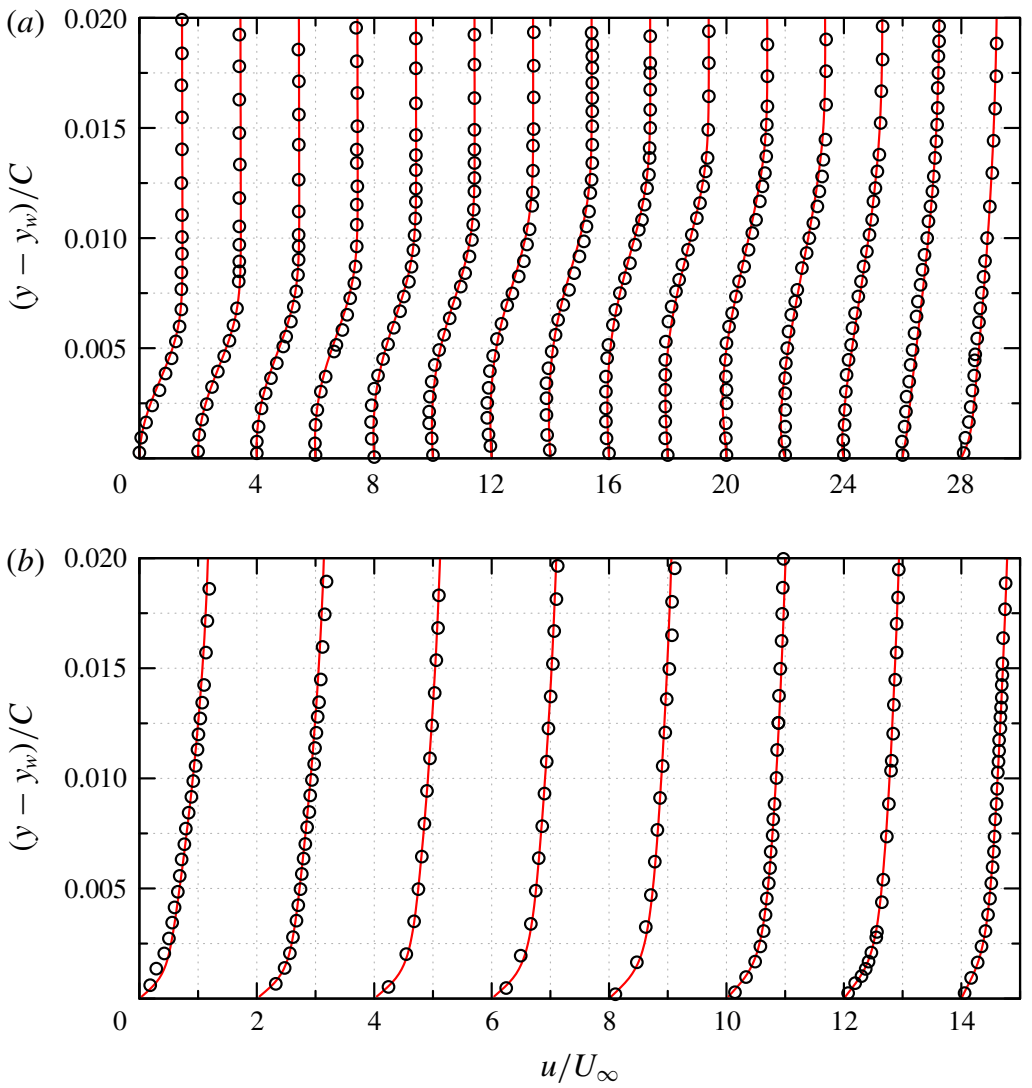

FIGURE 8. (Colour online) NACA0018, $R e_{c}=10^{5}$. The mean velocity profiles in the $x$-direction, $u$ along the vertical lines at different locations of the suction side. From left to right, (a) $x / \bar{C}=0.2-0.48$ with equal distances of 0.02 , shifted by $0,2, \ldots, 28$; (b) $x / \bar{C}=0.50,0.52,0.54,0.56,0.60,0.66,0.73,0.87$, shifted by $0,2, \ldots, 14$. present WMLES; O, experimental data from Kirk \& Yarusevych (2017) and Boutilier \& Yarusevych (2012).

figure $7 a$ ). This sharp drop may be explained by examining the LES results in detail: it is attributed to a separation bubble connected by turbulent transition as observed in the skin-friction plot in figure $7(b)$. It is reported in the experiment by Kirk \& Yarusevych (2017) that the onset of turbulent transition occurs at $x / C=0.44 \pm 0.02$, discerned from the dip in the $C_{p}$-profile. The WMLES shows larger fluctuations at locations $x / C \in[0.38,0.48]$ in comparison with the experiment, and the source of this difference remains to be explored further.

\subsection{Validation of WMLES: high $\operatorname{Re}_{c}$ case, $\operatorname{Re}_{c}=2.1 \times 10^{6}$}

The time- and spanwise-averaged $C_{f}$ and $C_{p}$ for the high Reynolds number case of the A-airfoil are shown in figure 10 for the present WMLES, the experiment by Gleyzes (1988) and also WRLES results by Mary \& Sagaut (2002) and Asada \& Kawai (2018). The pressure coefficient compares well with the experiment, and the separation near the trailing edge, as indicated by $C_{f}$-plot (see figure $10 b$ ), is also well captured by 

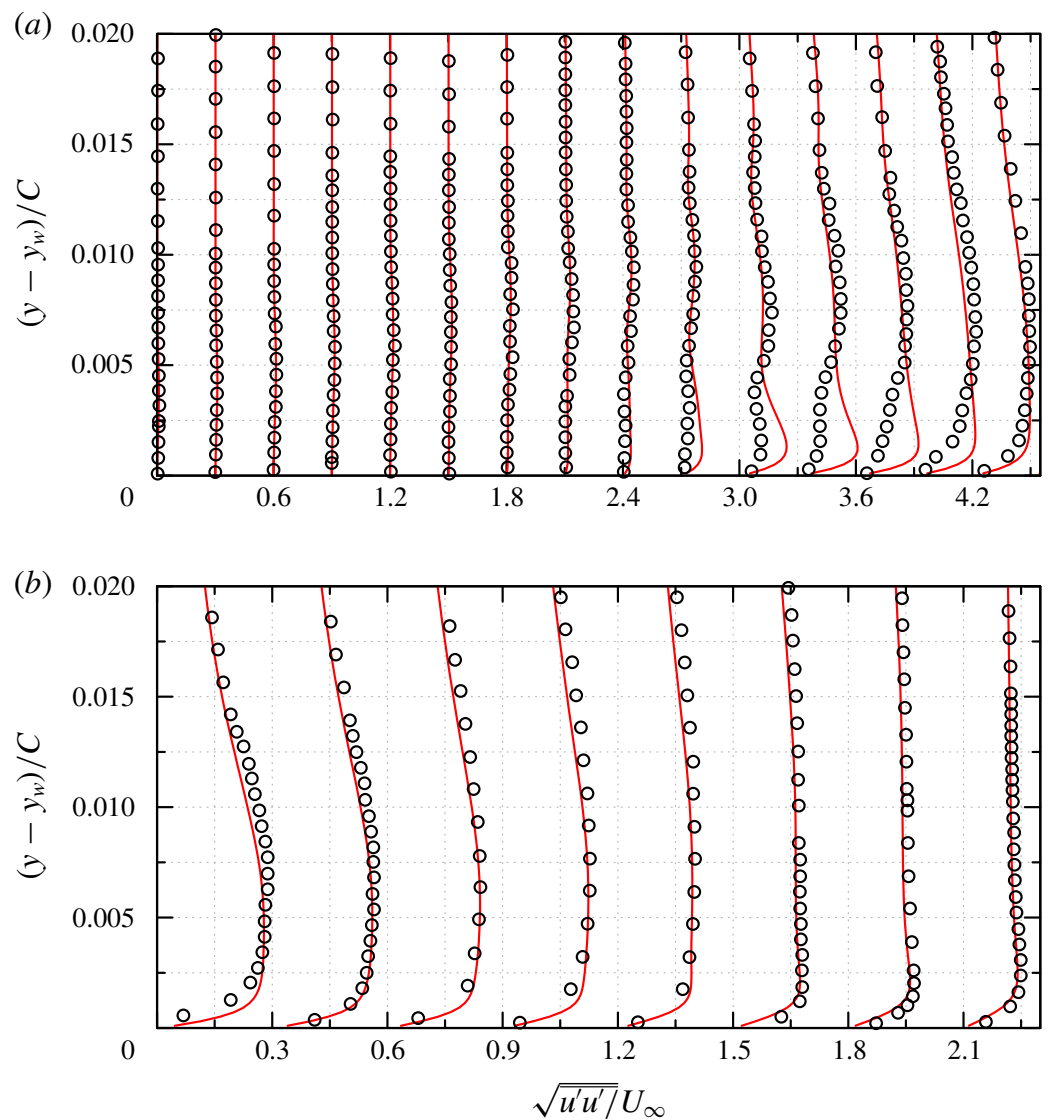

FIgURE 9. (Colour online) NACA0018, $R e_{c}=10^{5}$. The Reynolds stress profiles, $\sqrt{\overline{u^{\prime} u^{\prime}}} / U_{\infty}$ along the vertical lines at different locations of the suction side. From left to right, (a) $x / \bar{C}=0.2-0.48$ with equal distances of 0.02 , shifted by $0,0.3, \ldots, 4.2$; (b) $x / \bar{C}=0.50,0.52,0.54,0.56,0.60,0.66,0.73,0.87$, shifted by $0,0.3, \ldots, 2.1$. present WMLES; O, experimental data from Kirk \& Yarusevych (2017) and Boutilier \& Yarusevych (2012).

the present WMLES. The trailing edge separation occurs at approximately $x / \bar{C}=$ 0.90 compared with the experimental observation $(x / \bar{C} \approx 0.82)$ (Gleyzes 1988). In the experiment a tiny separation bubble close to the leading edge, which reattaches at $x / \bar{C}=0.12$ is observed. In the present WMLES, although instantaneous velocity contours clearly show a local reversal flow in this region, the time- and spanwiseaveraged $C_{f}$ does not capture this tiny separation bubble.

The streamwise mean velocity profiles on the suction side are shown in figure 11 . The flow reversal in the separation zone $(x / \bar{C}=0.93,0.99)$ is well captured by the WMLES. The Reynolds stress profiles corresponding to the root mean square (r.m.s.) streamwise fluctuations, wall-normal fluctuations and off-diagonal terms are shown in figure 12. Overall the WMLES predicted values of the Reynolds stress components are in general agreement and follow the trends in the experimental data. 

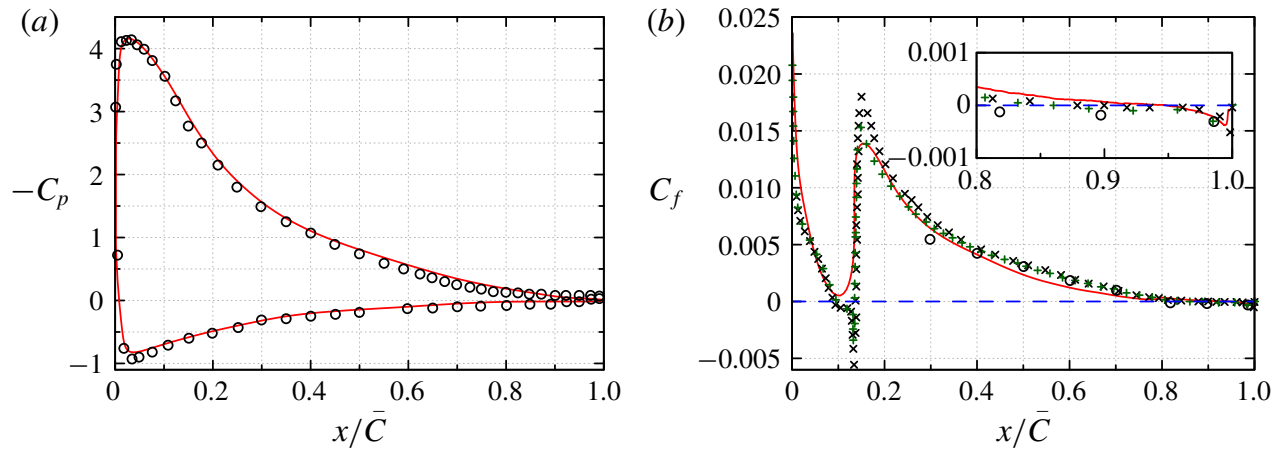

Figure 10. (Colour online) Aérospatiale A-airfoil, $R e_{c}=2.1 \times 10^{6}$. (a) Distribution of the pressure coefficient $C_{p}$, and $(b)$ skin-friction coefficient $C_{f}$ on the suction surface. O, experimental data from Gleyzes (1988); ×, WRLES from Mary \& Sagaut (2002); +, WRLES from Asada \& Kawai (2018); _ present WMLES. The - - - line $C_{f}=0$ is shown for convenience: zero crossing of this line indicates separation and reattachment.

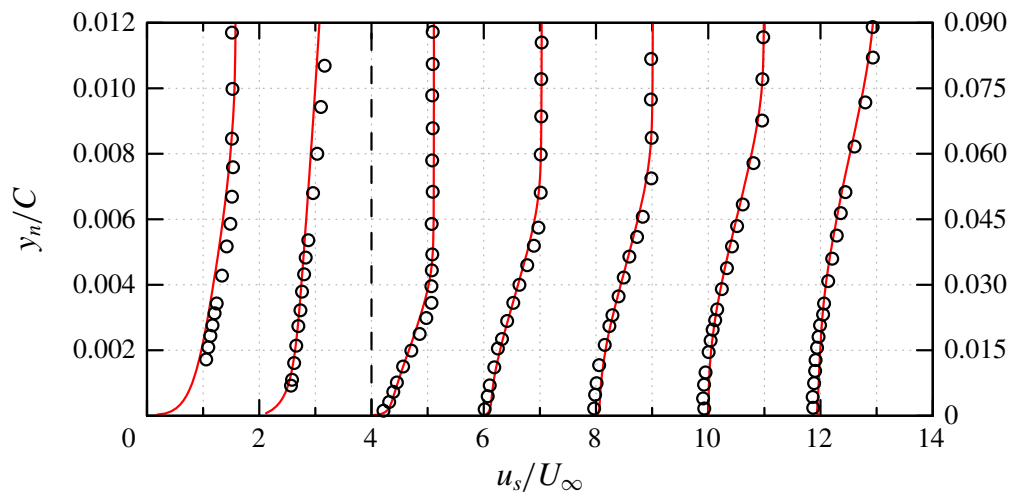

Figure 11. (Colour online) Aérospatiale A-airfoil, $R e_{c}=2.1 \times 10^{6}$. The mean streamwise velocity profiles, $u_{s}$ along the wall-normal lines at different locations of the suction side. From left to right, $x / \bar{C}=0.3,0.5,0.7,0.825,0.87,0.93,0.99$. Profiles are shifted by $0,2, \ldots, 12$ for clarity. $\bigcirc$, experimental data from Gleyzes (1988); — , present WMLES. Note that the profiles on the left-hand side of the vertical dashed lines use the left $y$-axis, while the others use the right $y$-axis.

\section{Anisotropy of the flow}

In the above section, we noted that the Reynolds stress components for all three WMLES cases (ranging from low to high $R e_{c}$ ) are found to be in reasonable agreement: the prediction location of transition onset is very close to DNS for $R e_{c}=10^{4}$, the WMLES results agree with experimental data by Boutilier \& Yarusevych (2012) and Kirk \& Yarusevych (2017) for $R e_{c}=10^{5}$ and follow the general trend of experiments by Gleyzes (1988) for $R e_{c}=2.1 \times 10^{6}$. It is, therefore, interesting to further analyse the LES data to recognize the flow patterns around the airfoil. The objective of the present section is to examine the anisotropy in these wall-bounded flows. 

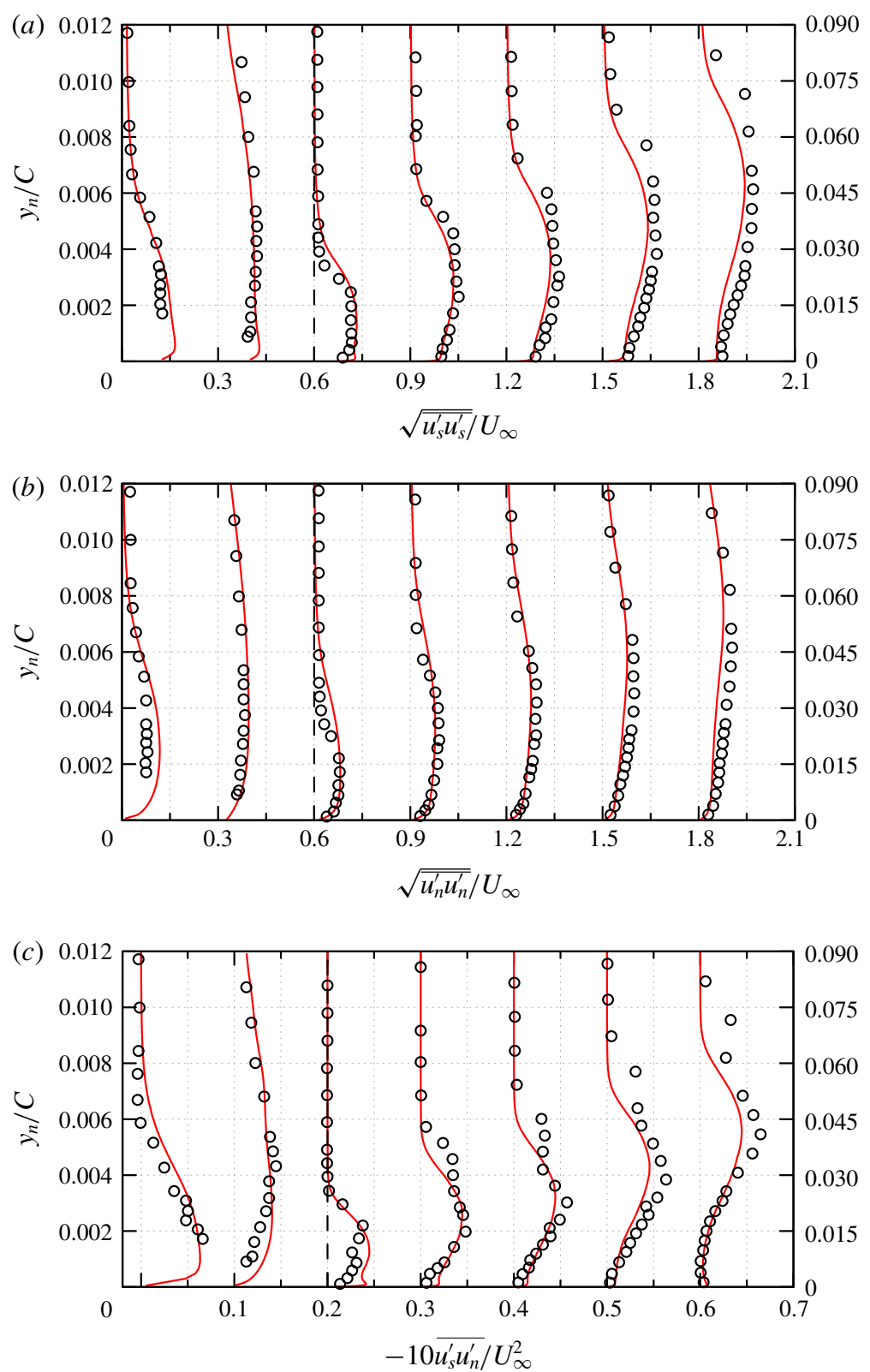

Figure 12. (Colour online) Aérospatiale A-airfoil, $R e_{c}=2.1 \times 10^{6}$. The Reynolds stress profiles, (a) $\sqrt{\overline{u_{s}^{\prime} u_{s}^{\prime}}} / U_{\infty}, \quad(b) \sqrt{\overline{u_{n}^{\prime} u_{n}^{\prime}}} / U_{\infty}$ and (c) $\overline{u_{s}^{\prime} u_{n}^{\prime}} / U_{\infty}^{2}$ along the wallnormal lines at different locations of the suction side. From left to right, $x / \bar{C}=$ $0.3,0.5,0.7,0.825,0.87,0.93,0.99$. Profiles are shifted by $0,0.3, \ldots, 1.8$ in $(a)$ and $(b)$, and shifted by $0,0.1, \ldots, 0.6$ in $(c)$ for clarity. $\bigcirc$, experimental data from Gleyzes (1988); - , present WMLES. 


\subsection{Definition of anisotropy}

The non-dimensional anisotropy tensor introduced by Lumley \& Newman (1977) and Lumley (1979) is defined as

$$
b_{i j}=\overline{u_{i}^{\prime} u_{j}^{\prime}} / \overline{u_{k}^{\prime} u_{k}^{\prime}}-\delta_{i j} / 3 .
$$

It has a zero trace $\left(b_{i i}=0\right)$ and is characterized by two invariants, viz.,

$$
I I=-b_{i j} b_{i j} / 2, \quad I I I=b_{i j} b_{j k} b_{k i} / 3,
$$

which allow a simple graphical evaluation, i.e. $(I I I, I I)$-plane, of possible states of turbulence. It is more convenient to identify the anisotropy by

$$
\phi=\left(\frac{I I I}{2}\right)^{1 / 3}, \quad \psi=\left(-\frac{I I}{3}\right)^{1 / 2}
$$

(Pope 2000). At any location in a turbulent flow, $\phi$ and $\psi$ are determined from the Reynolds stress components, which correspond to points in the $(\phi, \psi)$-plane, as shown in figure 13. All realizable states of the anisotropy tensor are found within a triangular region in the $(\phi, \psi)$-plane, which is the anisotropy invariant map (AIM) and often referred to as the Lumley triangle (figure 13 ). The origin of the triangle, i.e. $(0,0)$ corresponds to 3-D isotropic turbulence, the left-hand corner point of the triangle, i.e. $(-1 / 6,1 / 6)$ corresponds to two-component $(2 \mathrm{C})$ isotropic turbulence, the righthand corner point of the triangle, i.e. $(1 / 3,1 / 3)$ corresponds to one-component (1C) turbulence. The turbulence along the upper line connecting the $2 \mathrm{C}$ isotropic turbulence and $1 \mathrm{C}$ turbulence is the $2 \mathrm{C}$ turbulence state, in which

$$
\psi=\left(\frac{1}{27}+2 \phi^{3}\right)^{1 / 2} .
$$

The left-hand line $\psi=-\phi$ and right-hand line $\psi=\phi$ correspond to the 'axisymmetric contraction' and 'axisymmetric expansion' state, respectively (Choi \& Lumley 2001). This classification of turbulence is formally based on the shape of the energy ellipsoid, and details of the analysis may be obtained from Lumley (1979).

The above anisotropy invariants find many uses in the turbulence modelling community. First, they help to define realizable states of the Reynolds stress tensor, i.e. all physically realizable turbulence is bounded in the AIM. Second, it is desirable to ensure that the simulated turbulence field can only pass through a succession of realizable states, which helps improve the anisotropy-resolving turbulence closures especially at second-moment level. Furthermore, the invariants should satisfy some specific relations or bounds at the limiting states.

\subsection{Low Reynolds number $R e_{c}=10^{4}$ case}

Figures 13 (WMLES) and 14 (DNS) show the loci associated with traversals in the vertical direction at six locations $(x / C=0.8,0.9,0.92,0.94,0.96,0.98)$ along the suction surface of the NACA0012 airfoil. Overall, the loci behave similarly in both WMLES and DNS at the same locations. It is observed that all states indeed lie within the triangle, which is required by realizability constraints. For $x / C=0.8$ as shown in figures 13(a) and 14(a), all the turbulence states are concentrated at the right corner of the Lumley triangle. This indicates that most of the turbulence at this location, before the transition onset occurs (see figure 6), corresponds to $1 \mathrm{C}$ and $2 \mathrm{C}$ turbulence. 


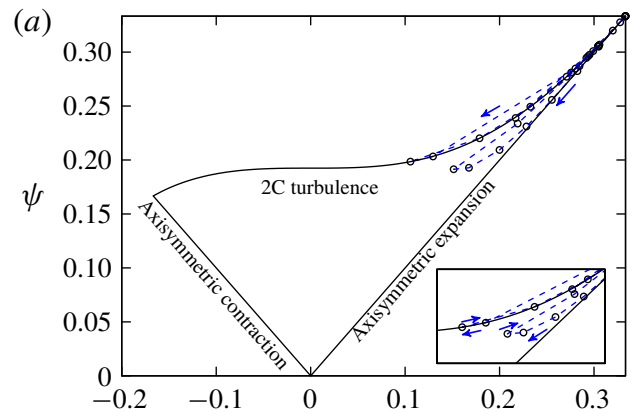

(b)
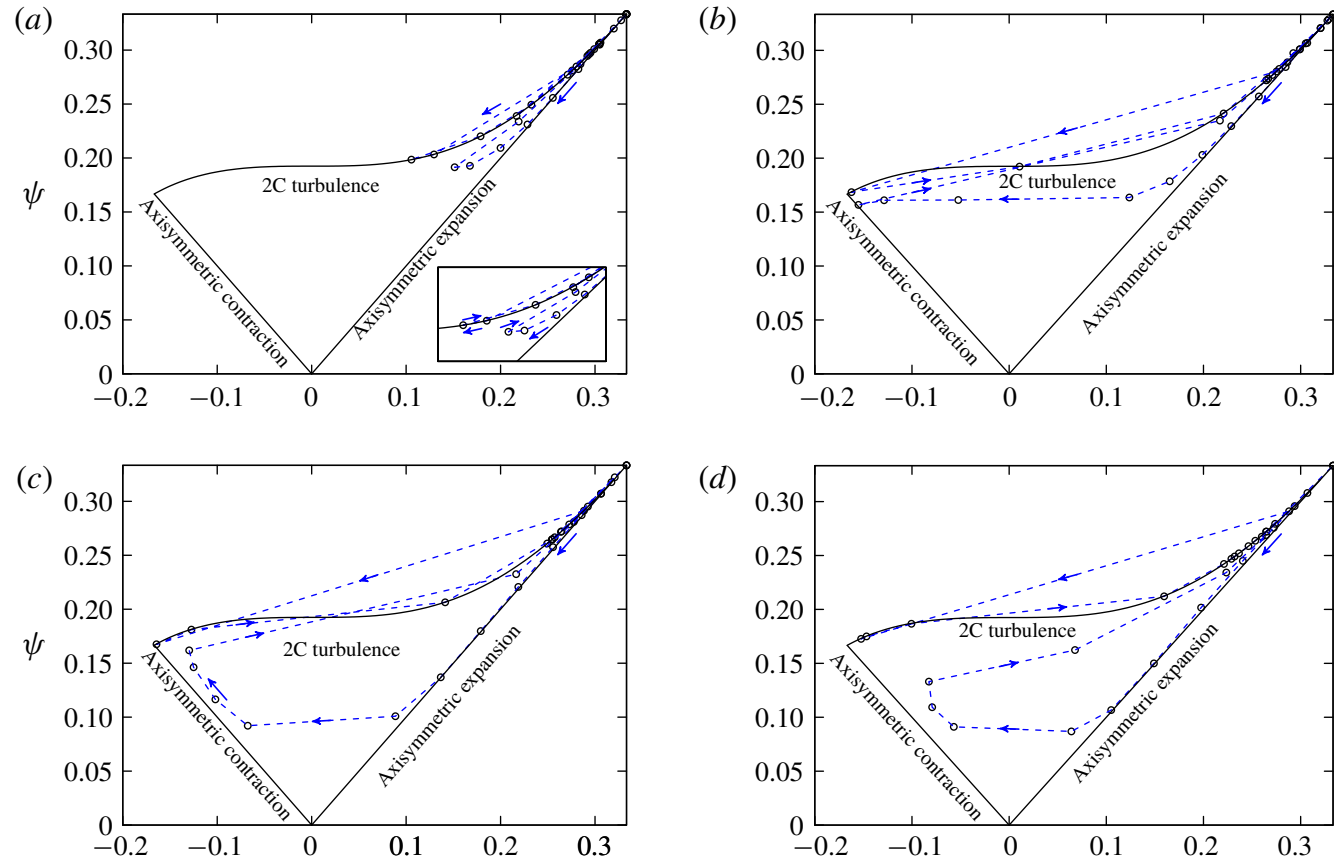

(d)
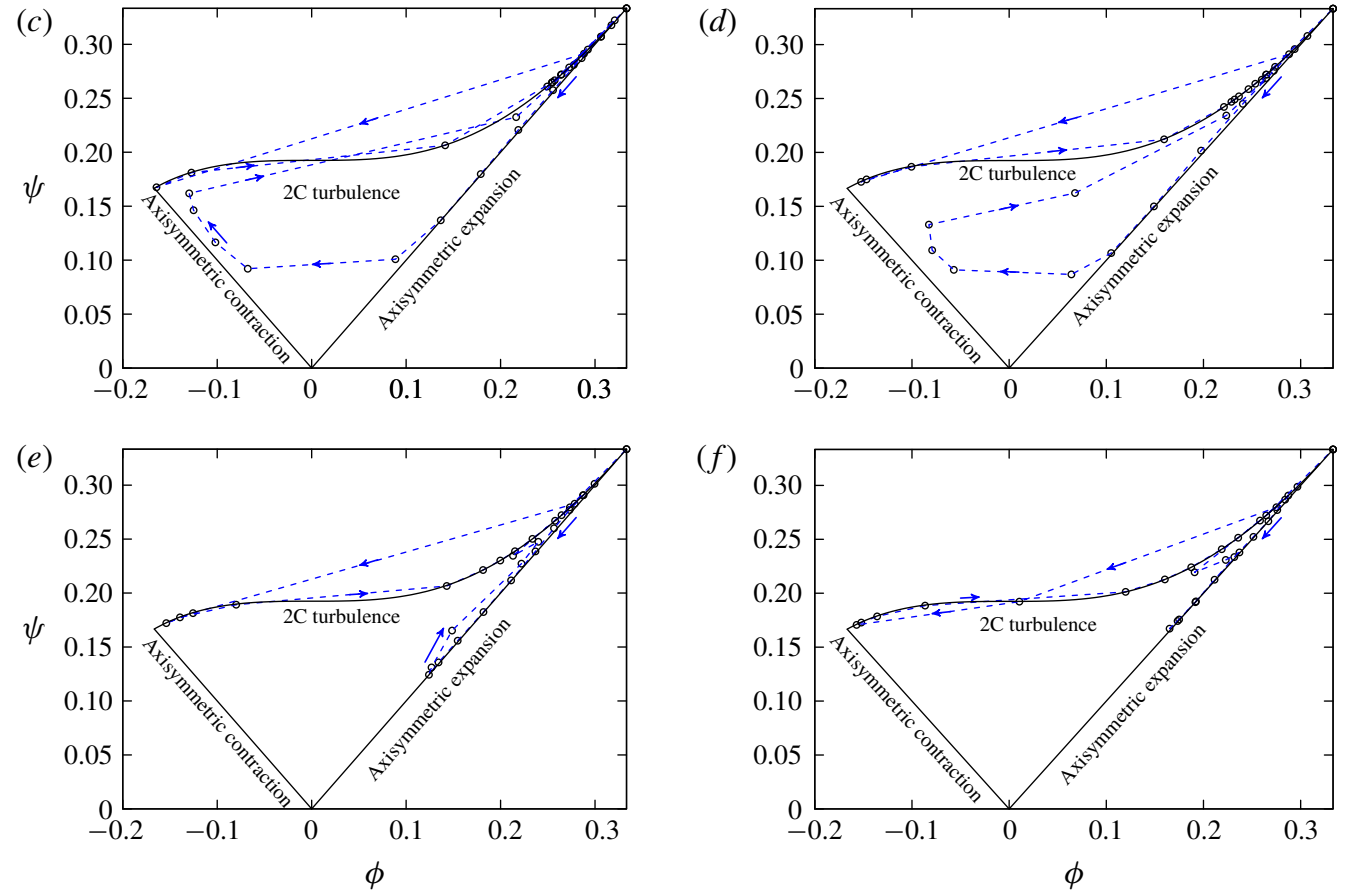

FIgURE 13. (Colour online) NACA0012, $R e_{c}=10^{4}$. Invariant maps of WMLES results along vertical lines at six locations along the suction surface: $(a) x / C=0.8 ;(b) x / C=0.9$; (c) $x / C=0.92 ;(d) x / C=0.94 ;(e) x / C=0.96 ;(f) x / C=0.98 . \longrightarrow$ denotes the direction away from the airfoil surface. The line partly outside the triangle at the upper boundary is due to the connection of two neighbouring points.

For the near-wall region, the turbulence state is seen to approach the two-component state at all six locations and then progresses along the 'axisymmetric expansion' line (similar to that in the log region of a channel flow (Pope 2000)) and ending in the upper portion of the 'two-component turbulence' line. However, the manner in which this process takes place varies at different locations. Within the separation zone close to the reattachment point, $x / C=0.92$ and $x / C=0.94$, some points are close to the 'axisymmetric contraction' line, similar to observations in a developed free shear layer (Bell \& Mehta 1990). For flow close to the trailing edge $(x / C=0.96$ and $x / C=$ 0.98), the upper-right pointing arrow along the 'axisymmetric expansion' line (log-law region) signifies a state very different from that in a free shear layer. 


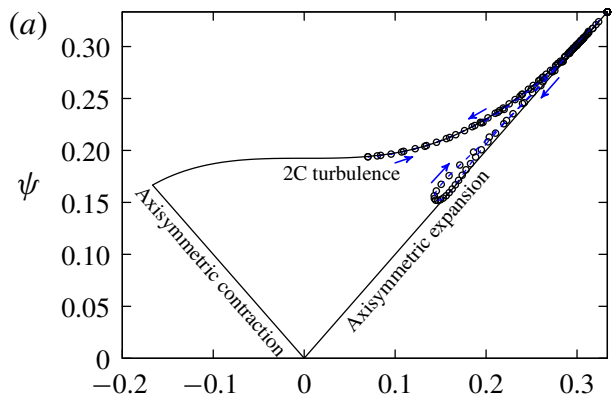

(b)

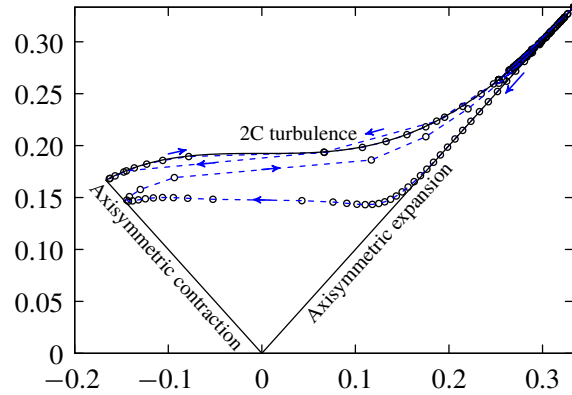

(c)

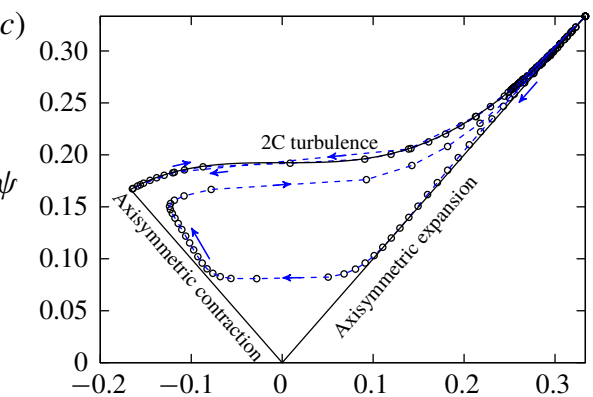

(d)
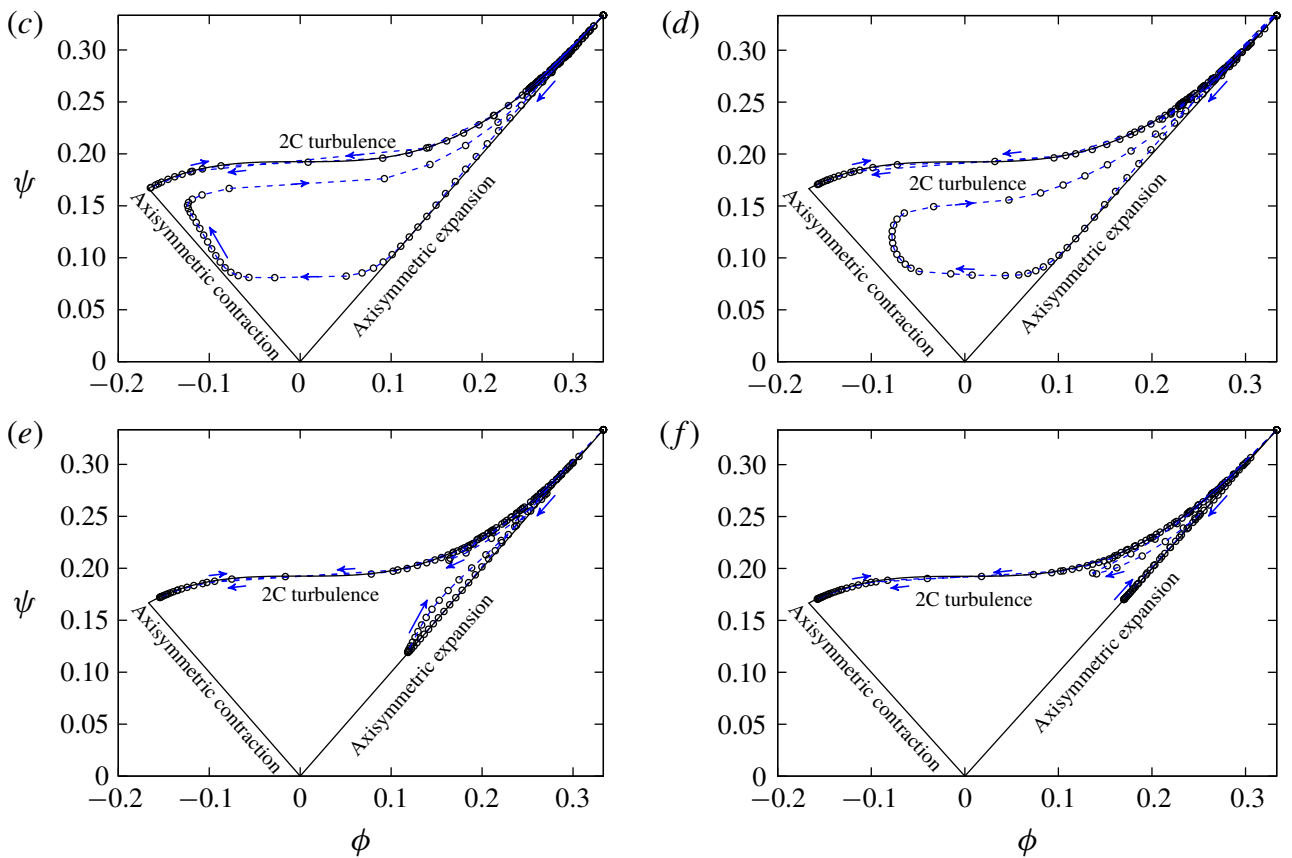

FIgURE 14. (Colour online) NACA0012, $R e_{c}=10^{4}$. Invariant maps of DNS results along vertical lines at six locations along the suction surface: $(a) x / C=0.8$; $(b) x / C=0.9$; (c) $x / C=0.92 ;(d) x / C=0.94 ;(e) x / C=0.96 ;(f) x / C=0.98 . \longrightarrow$ denotes the direction away from the airfoil surface. The line partly outside the triangle at the upper boundary is due to the connection of two neighbouring points.

\subsection{Moderate Reynolds number $R_{c}=10^{5}$ case}

Figure 15 shows the loci associated with traversals in the vertical direction at six locations $(x / C=0.4,0.45,0.5,0.65,0.8,0.95)$ along the suction surface of the NACA0018 airfoil. Substantially, the state of turbulence follows the same locus as observed in the NACA0012 case. A point of difference is that most turbulence states are concentrated on the 'two-component turbulence' line in the NACA0012 case, while most points concentrate near the 'axisymmetric expansion' and 'axisymmetric contraction' lines except for the flow in the separation zone $(x / C=0.4)$. This is very similar to the observation in the post-recirculation zone of channel flow with periodic hills at $R e_{h}=10595$ (Fröhlich et al. 2005). The backward-moving shear flow in the separation zone behaves similarly to a thin boundary layer as the wall is approached, 

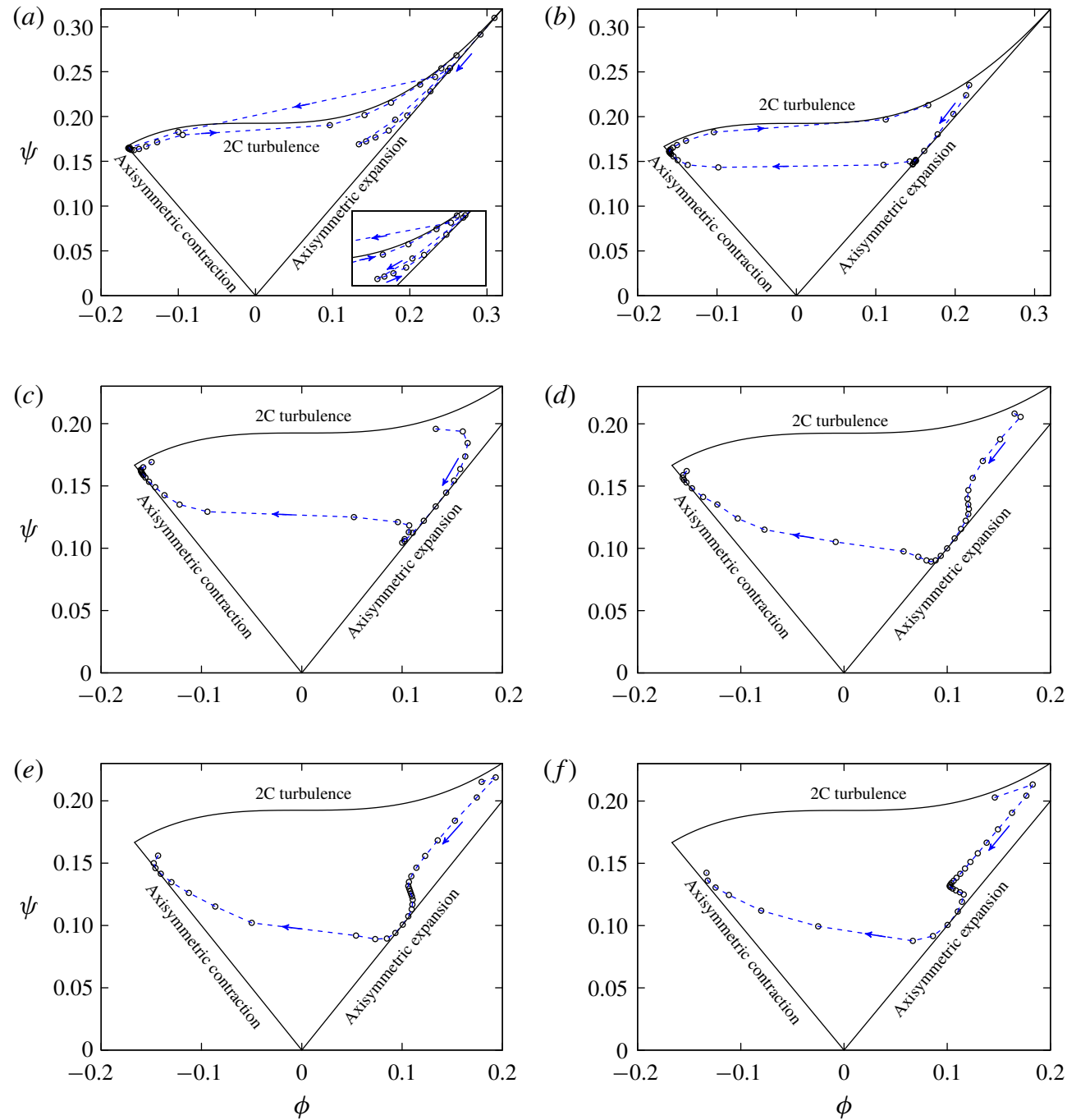

FIgURE 15. (Colour online) NACA0018, $R e_{c}=10^{5}$. Invariant maps of WMLES results along vertical lines at six locations along the suction surface: $(a) x / C=0.4 ;(b) x / C=$ $0.45 ;(c) x / C=0.5 ;(d) x / C=0.65 ;(e) x / C=0.8 ;(f) x / C=0.95$. $\longrightarrow$ denotes the direction away from the airfoil surface. The line partly outside the triangle at the upper boundary in $(a)$ is due to the connection of two neighbouring points.

and thus the flow structure has to approach the two-component limit at the wall. After the separation bubble, the shear layer develops, with more points concentrated on the 'axisymmetric contraction' line after the log-law region.

\subsection{High Reynolds number $\operatorname{Re}_{c}=2.1 \times 10^{6}$ case}

Figure 16 shows the loci associated with traversals in the vertical direction at eight locations $(x / C=0.35,0.55,0.7,0.8,0.85,0.90,0.94,0.96)$ along the suction surface of the A-airfoil. The loci vary significantly at different locations. In the attached 
(a)

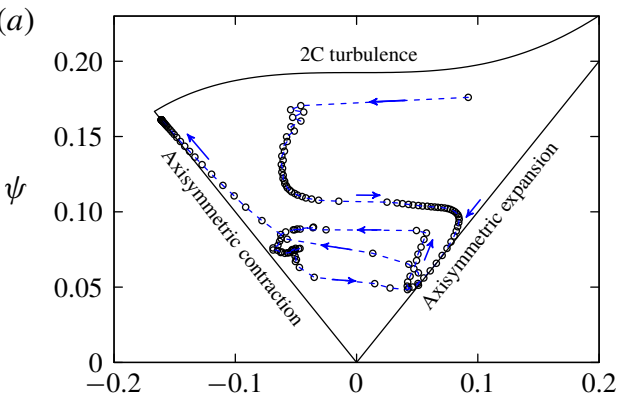

(c)

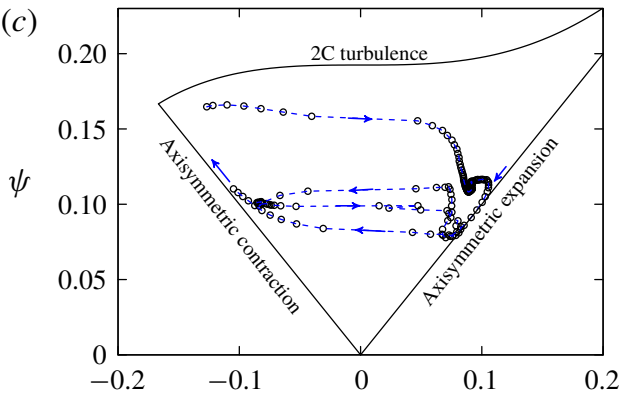

(e)

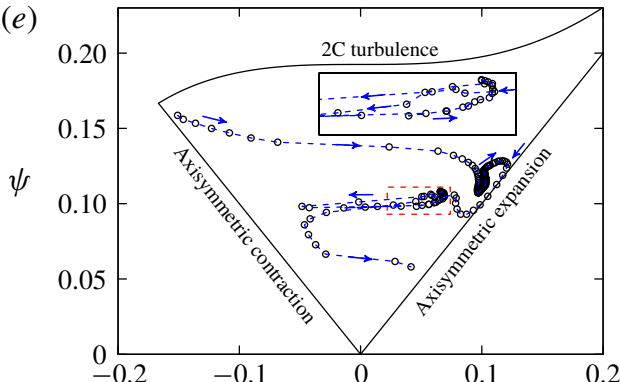

$(g)$

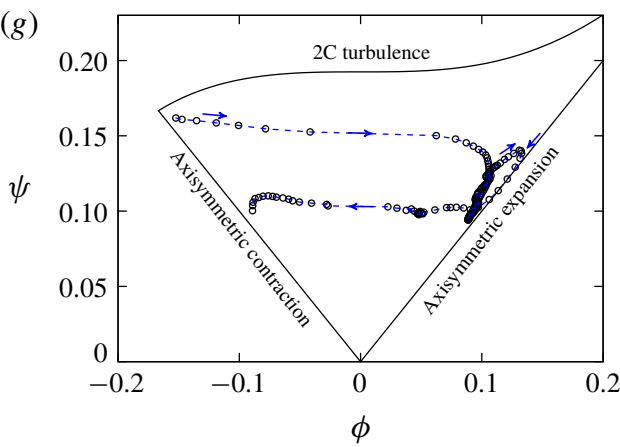

(b)

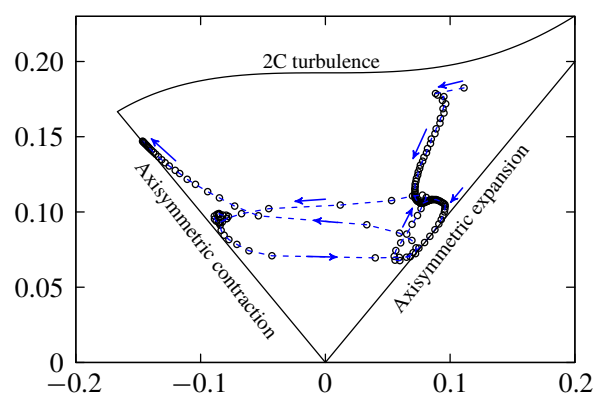

(d)

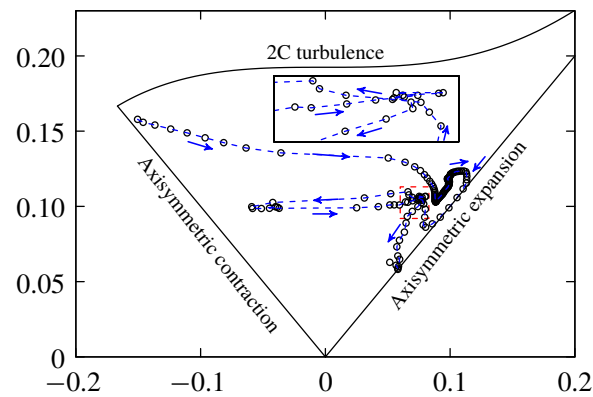

(f)

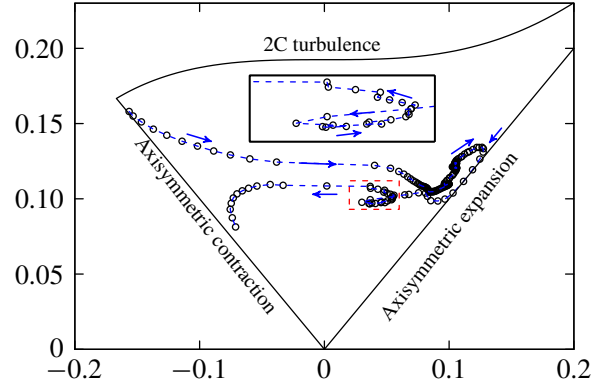

(h)

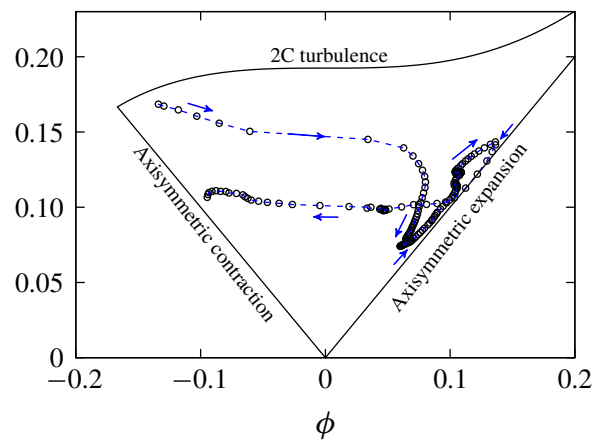

FIGURE 16. (Colour online) A-airfoil, $R e_{c}=2.1 \times 10^{6}$. Invariant maps of WMLES results along vertical lines at eight locations along the suction surface: $(a) x / C=0.35 ;(b) x / C=$ $0.55 ;(c) x / C=0.70 ;(d) x / C=0.80 ;(e) x / C=0.85 ;(f) x / C=0.90 ;(g) x / C=0.94$; (h) $x / C=0.96$. $\longrightarrow$ denotes the direction away from the airfoil surface.

zone, $x / C=0.35,0.55,0.7,0.8,0.85$, the locus of the turbulence state follows a similar approach beyond the wall, i.e. from $2 \mathrm{C}$ turbulence to log-law region, and 
then free shear-layer region. As the location moves closer towards the separation point fewer points concentrate along the 'axisymmetric contraction' line. In the recirculation zone, $x / C=0.9,0.94,0.96$, most of the states are clustered along the 'axisymmetric expansion' line although a portion of the locus approaches the 'axisymmetric contraction' line. In the present case, the anisotropy in a free shear layer is considerably lower than that in a near-wall layer, and thus the turbulence state never approaches the two-component state at the end.

\section{Unsteady flow separation and reattachment}

It is evident that the time- and spanwise-averaged skin friction (i.e. $C_{f}$-plot) at the airfoil surface provides much useful information for interpreting separation and reattachment behaviour. However, these mean-flow concepts are insufficient to fully understand the flow physics in its entirety including the unsteady force load and temporal turbulent transition that are seen in these aerodynamic flows. Such unsteady flow behaviour manifested near the trailing edge may result in detrimental phenomena such as airfoil vibration. In the present section, we attempt to understand the unsteady flow behaviour through a presentation of skin-friction lines, i.e. curves tangent to the local skin-friction vector and interpreted as limiting streamlines for the near-wall flow. We also use the plot of the spanwise-averaged flow field to illustrate the skin-friction lines.

We briefly digress to discuss flow past circular smooth, and grooved cylinders referring to the recent work by Cheng et al. (2017, 2018a). In this series of study, the sudden change of aerodynamic force (the drag crisis) around the cylinder is ascribed to the unsteady interaction of primary separation and secondary separation behaviour. This unsteady separation interaction effect and the turbulent transition effect both appear in the drag crisis of flow past a smooth cylinder. On the other hand, for the grooved cylinder the unsteady separation interaction effect still dominates the flow and results in a similar drag crisis phenomenon, but the turbulent transition effect is difficult to discern. In the present study of flow past airfoils, we note that the airfoil itself has smoothly varying curvature along its surface ranging from large curvature near the stagnation point to zero curvature near the trailing edge (excluding the sharp trailing edge point). While the airfoil is clearly different from a smooth cylinder of constant curvature, and also different from a grooved cylinder with larger curvature change over short distances, it is nonetheless interesting and instructive to examine the instantaneous flow field. The discussion presented here focuses on time sequences of the flow and examines the correspondences between the spanwise-averaged streamlines, the spanwise-averaged skin-friction coefficient $C_{f}$ and the surface streamlines on the upper or suction side of the airfoil under consideration.

Analysing the unsteady flow patterns is a challenging task owing to the vast amount of data generated in these unsteady 3-D simulations. A rational approach is warranted to identifying key time instants where the flow separation behaviour shows significant differences. Here we use a time evolution instantaneous $\tilde{u}(t) / U_{\infty}$ for all three cases at specified monitoring points (see figure 17) to aid the selection of specific time instances for a closer examination of surface streamlines (discussed in the ensuing sub-sections).

\subsection{Data analysis for three cases}

For the NACA0012 airfoil, the monitoring point is located at $x / C=0.96$ inside the separation zone. As shown in figure 17(a), which shows a somewhat regular 

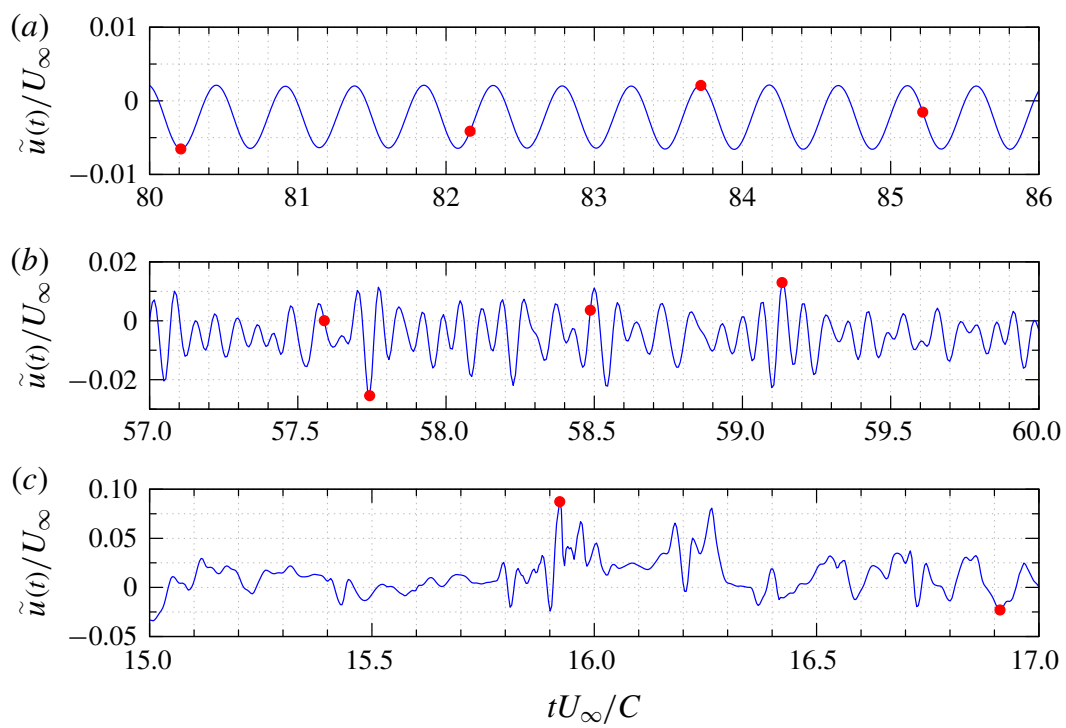

FIgURE 17. (Colour online) Time history of $\tilde{u}(t) / U_{\infty}$ at the first grid point at specified streamwise locations. (a) NACA0012, $R e_{c}=10^{4}, x / C=0.96 ;(b)$ NACA0018, $R e_{c}=10^{5}$, $x / C=0.35$; $(c)$ Aérospatiale A-airfoil, $R e_{c}=2.1 \times 10^{6}, x / C=0.882$. The marked points denote the selected time instances for analysing the unsteady separation behaviour in the near-wall regions.

oscillation, four typical instantaneous snapshots of the near-wall flow field are chosen to analyse the surface skin-friction profiles: two corresponding to a local maximum and minimum, and one each corresponding to the upward and downward flow speed.

For the NACA0018 airfoil, where the flow is still regular but with more than one dominant frequency, the monitoring point is located at $x / C=0.35$. Four typical instantaneous snapshot of the near-wall flow field are adopted, as shown in figure $17(b)$, similar to the choice of the NACA0012 airfoil.

For the A-airfoil, the monitoring point is located at $x / C=0.882$. Only two typical instantaneous snapshot of the near-wall flow field are chosen: one at a local maximum and the other at a local minimum (see figure $17 c$ ), due to strongly random variations of $\tilde{u}(t) / U_{\infty}$.

For every time instant analysed in the next several plots, we use the spanwiseaveraged wall-parallel skin-friction coefficient $C_{f}$, spanwise-averaged streamlines and skin-friction lines of the instantaneous skin-friction vector $\boldsymbol{C}_{\boldsymbol{f}}$. These include figure 18 for the case of $R e_{c}=10^{4}$, figure 19 for the case of $R e_{c}=10^{5}$ and figure 20 for the case of $R e_{c}=2.1 \times 10^{6}$.

In every plot, we show a dashed line to represent every zero-crossing point in the spanwise-averaged $C_{f}$ plot, and label it as $S_{i}$ if the flow is separating or $R_{i}$ if reattaching. It should be emphasized that, due to the prevalence of many local separation/reattachment bubbles, sometimes it is difficult to recognize the separation point and reattachment for a given specific bubble and thus the subscript is not exactly related to a fixed bubble. We label the subscript $i$ only for counting the critical points along the flow direction. 


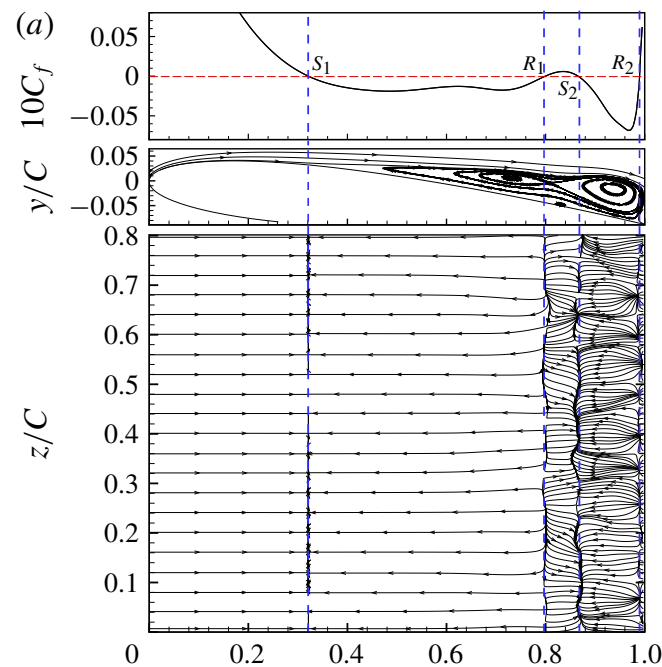

(b)
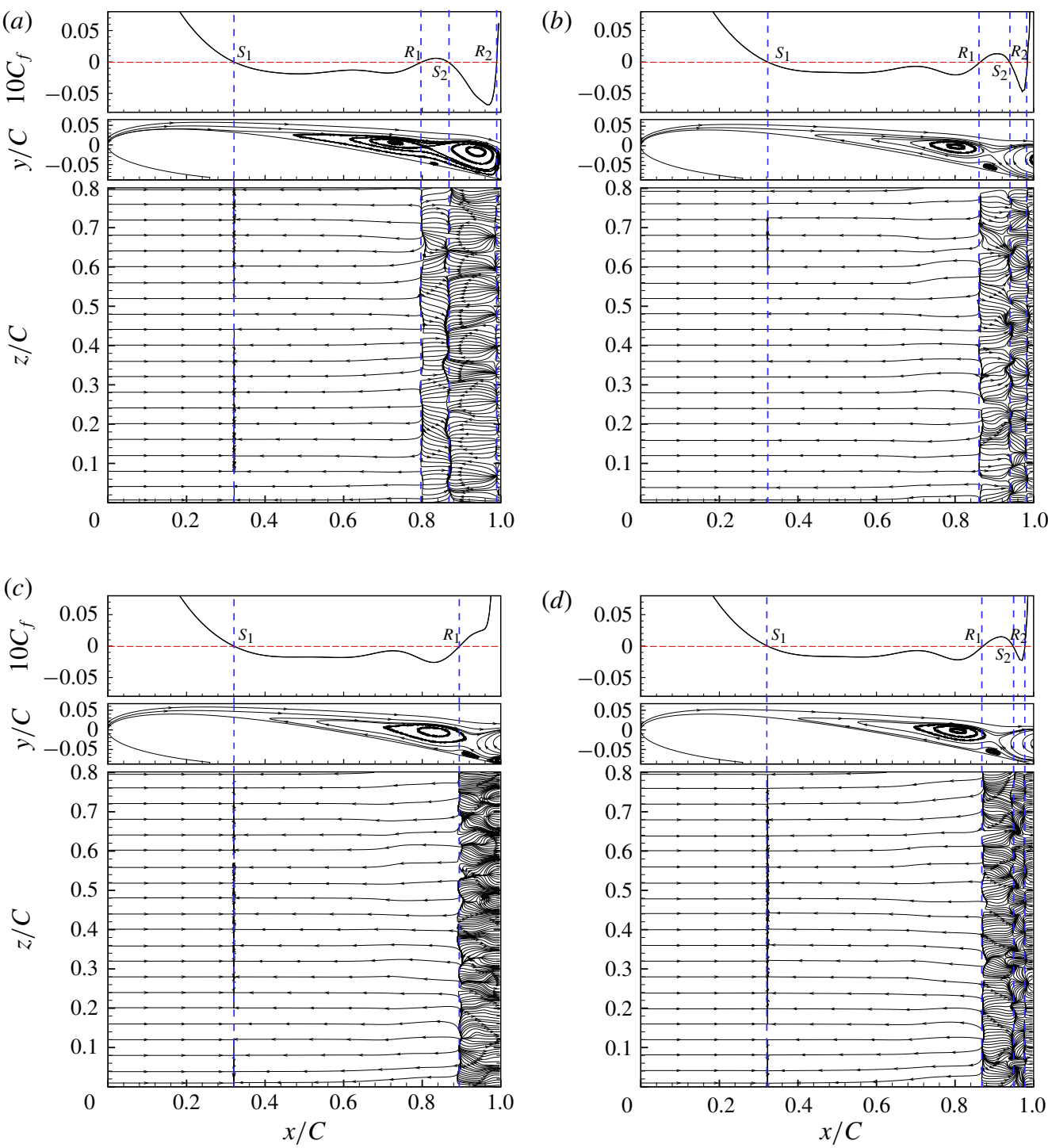

$(d)$

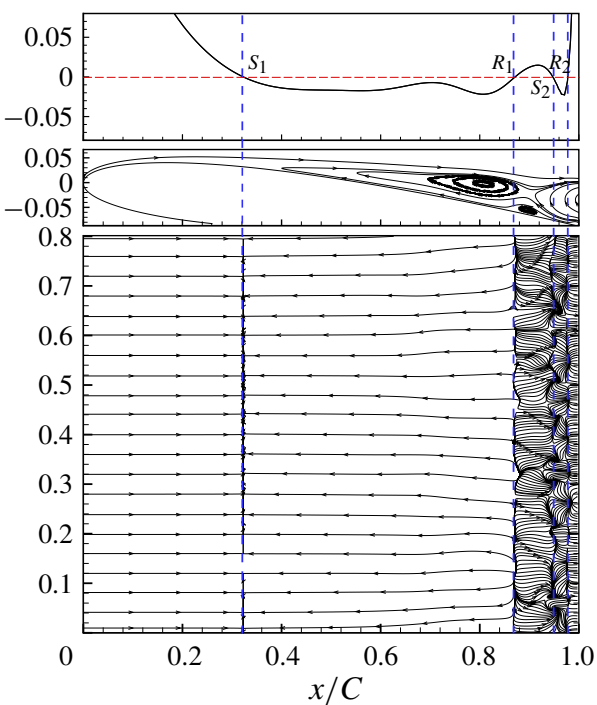

FIgURE 18. (Colour online) NACA0012, $R e_{c}=10^{4}$. The separation behaviour on the suction side at different instants. (a) $t=80.21 C / U_{\infty} ;$ (b) $t=82.16 C / U_{\infty} ;$ (c) $t=$ $83.72 C / U_{\infty} ;(d) t=85.22 C / U_{\infty}$. For each panel: top, the instantaneous spanwise-averaged friction coefficient $C_{f}$; middle, streamlines of the instantaneous spanwise-averaged flow filed; bottom, the skin-friction trajectories.

\section{2. $R e_{c}=10^{4}$}

In $\S 5.1$ where the WMLES results are verified against DNS of the NACA0012 case at $R e_{c}=10^{4}$, we observe shear layer turbulent transition at approximately $x / C=0.92$, which is very close to the trailing edge. In figure 18, there is a primary separation point, denoted $S_{1}$ in all the figures at $x / C=0.32$ : this is identified as the first zero crossing of $C_{f}$ and remains virtually unchanged for the entire time sequence. The correspondence between the spanwise-averaged streamlines, $C_{f}$ and surface streamlines, marked by a dashed blue line, is clear. Moreover, in the neighbourhood 

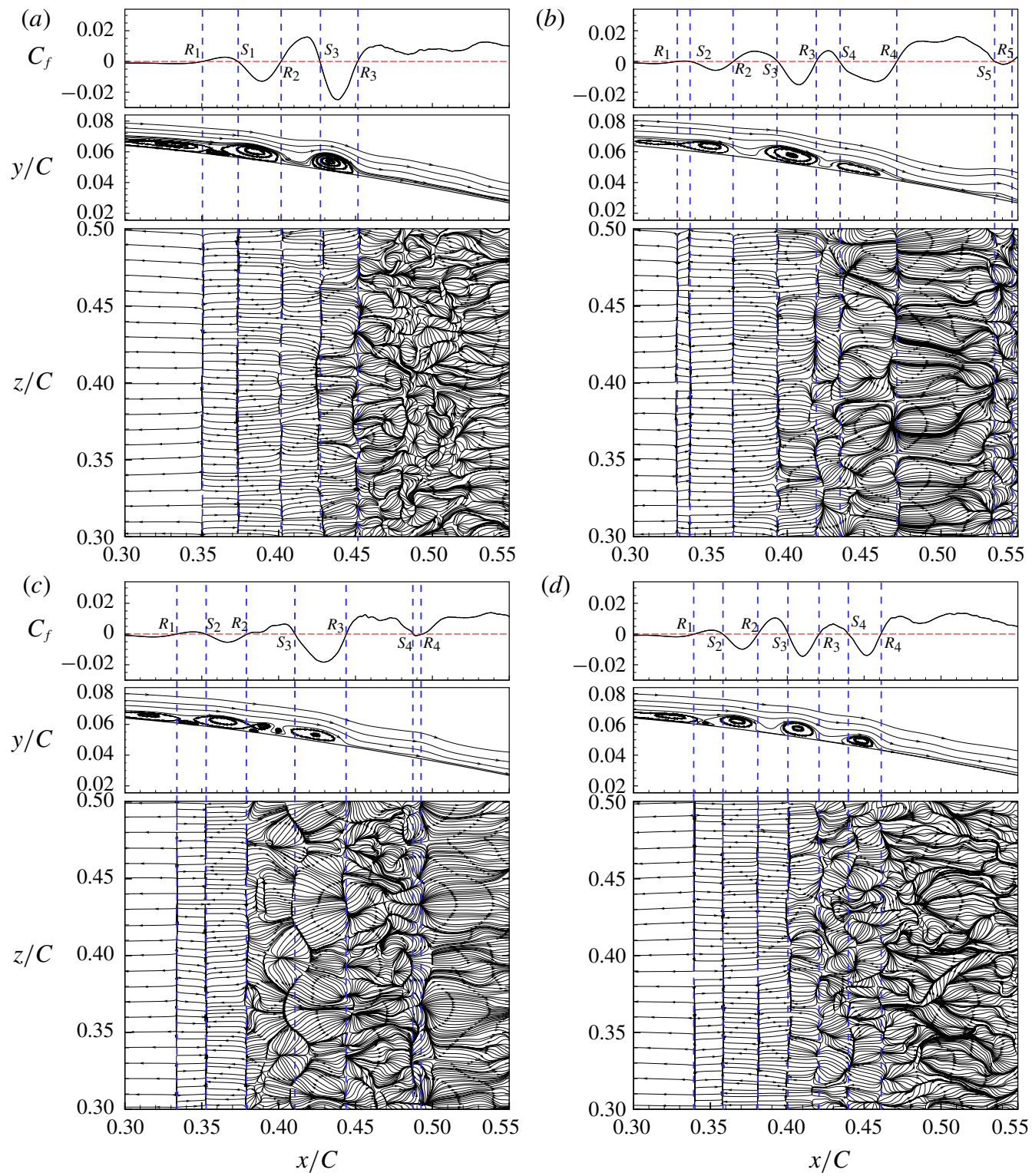

FIgURE 19. (Colour online) NACA0018, $R e_{c}=10^{5}$. The separation behaviour on the suction side at different instants: (a) $t=57.59 C / U_{\infty} ;(b) t=57.74 C / U_{\infty} ;$ (c) $t=$ $58.49 C / U_{\infty} ;(d) t=59.13 C / U_{\infty}$. For each panel: top, the instantaneous spanwise-averaged friction coefficient $C_{f}$; middle, streamlines of the instantaneous spanwise-averaged flow filed; bottom, the skin-friction trajectories.

of the separation line on the airfoil surface, the surface streamlines are straight with no spanwise components, and converge at $x / C=0.32$. This is indicative of the fact that the flow is virtually two-dimensional with no spanwise variation until the primary separation line $S_{1}$. This separation point $S_{1}$ marks the beginning of the globally separated flow, and constitutes the 'primary separation bubble' (PSB). This bubble reattaches near the trailing edge, at point $R_{2}$ in figure $18(a, b, d)$, but not in figure $18(c)$. 


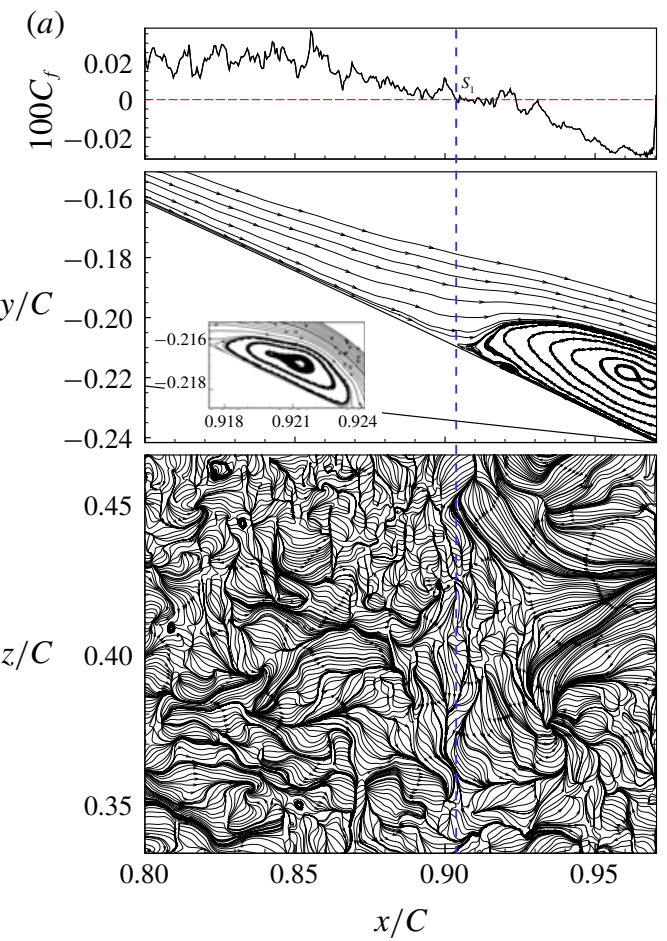

(b)
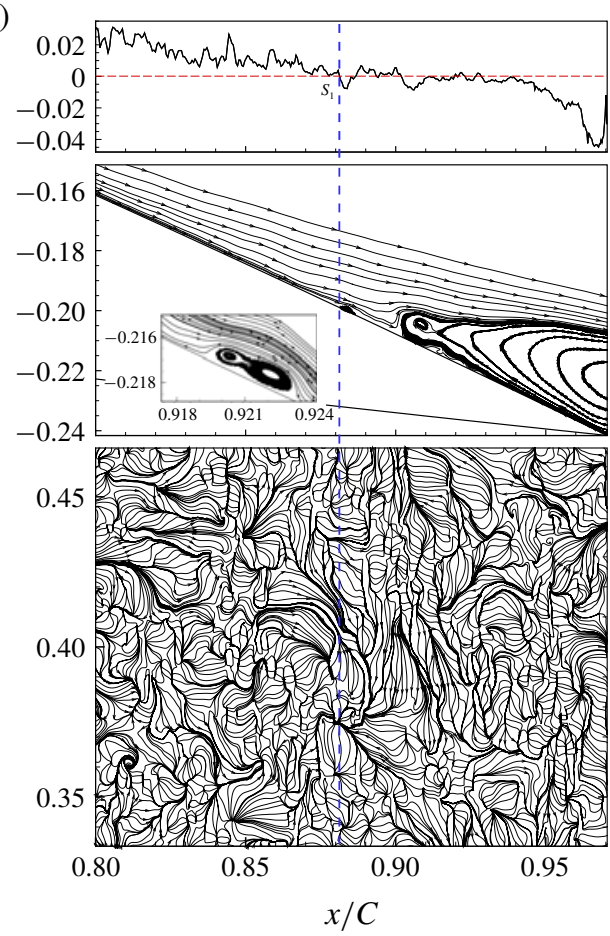

FIgure 20. (Colour online) A-airfoil, $R e_{c}=2.1 \times 10^{6}$. The separation behaviour on the suction side at different instants. (a) $t=15.92 C / U_{\infty} ;(b) t=16.91 C / U_{\infty}$. For each panel: top, the instantaneous spanwise-averaged friction coefficient $C_{f}$; middle, streamlines of the instantaneous spanwise-averaged flow filed; bottom, the skin-friction trajectories.

Inside the PSB, the flow shows a small secondary separated zone which separates at $S_{2}$ and reattaches at $R_{1}$. On surface streamline plots, these near-wall separation and reattachment flows degenerate to bundles of diverging/converging streamlines. This secondary separation bubble is also observed in figure $18(a, b, d)$, while it is not observed in $18(c)$. It is clear that during the shedding process, part of the vorticity is extracted from the primary separation bubble and the secondary separation bubble. We note that the separation points $\left(S_{2}\right)$ of the secondary separation bubble, if it exists, remain at around $x / C=0.87,0.94,0.96$; points which are close to the observed turbulent transition point through $-\overline{u^{\prime} v^{\prime}}$. From this viewpoint, it is plausible to conclude the secondary separation bubble is related to shear-layer turbulent transition. Instead of considering the secondary separation bubble as a signature of turbulent transition, we prefer to suggest that the wall-attached bubble is a source of perturbations leading to the shear layer turbulent transition.

$$
\text { 7.3. } R e_{c}=10^{5}
$$

Based on the time- and spanwise-averaged $\left(C_{p}, C_{f}\right)$ as plotted in figure 7 for the NACA0018 airfoil case, the turbulent boundary layer flow forms starting at approximately $x / C=0.45$. This turbulent boundary layer remains generally attached until the trailing edge. For this case, we mainly discuss the region of the transition part, which corresponding to the region $x / C \in[0.3,0.55]$ on the suction side of the airfoil, as shown in each plot in figure 19 . We note the separation point denoted $S_{1}$, which is close to the leading edge, is not visible in the present plots. 
A notable difference of the present case from the case of $R e_{c}=10^{4}$ is the disappearance of the primary separation bubble. For the NACA0018 case at $R e_{c}=10^{5}$, the flow exhibits strong unsteadiness, and the separated flow somewhat breaks up into several small bubbles. Among the four plots of figure 19, we can see points of $R_{1}, S_{2}, R_{2}, S_{3}$ and $R_{3}$ at all instants, but points $S_{4}, R_{4}, R_{5}$ and $R_{5}$ are seen only at some instants. Hence for this case, the interaction of several separation bubbles contributes to the shear-layer turbulent transition, and the flow, while more complex, may be considered as an extension of the mechanism in the case of $R e_{c}=10^{4}$ for NACA0012.

Another interesting phenomenon for this case at $R e_{c}=10^{5}$ is the spanwise length scale. In figure 19(c), features with a length scale of a fraction of the spanwise domain size are observed in the plot of skin-friction lines. While the flow remains two-dimensional up to point $R_{2}$ at approximately $x / C=0.38$, the spanwise variation beyond that point grows and a somewhat periodic behaviour in the spanwise direction is observed. At $x / C \approx 0.41$, structures with a scale of approximately $0.08 C$ are noted. These structures keep breaking into small-scale structures in the downward region, until reaching the turbulent region. Here we briefly comment on our somewhat larger spanwise domain extent compared with other airfoil simulations in the literature. From the unsteady and irregular surface flow patterns in the higher Reynolds number case, we point out that airfoil simulations employing small spanwise domain sizes may not be able to faithfully reproduce the unsteady flow patterns observed in our LES.

$$
\text { 7.4. } R e_{c}=2.1 \times 10^{6}
$$

Flow at $R e_{c}=2.1 \times 10^{6}$ endures not only shear-layer turbulent transition and corresponding attached turbulent boundary layer flow (shear-layer development and log-law region, see figure 16), but also turbulent separation, which is of interest here. In figure 20, we use data at two instants and plot the spanwise-averaged skin-friction coefficient, spanwise-averaged streamlines and surface streamlines, focusing on the turbulent separation region of $x / C \in[0.8,1.0]$.

Skin-friction lines at this high Reynolds number show features of a distinct length scale. In both plots, we can observe two distinct flows, with some large-scale structures corresponding to typical turbulent flow, and some small-scale structures which are essentially local separation/reattachment cells and are signatures of local flow reversals. The aggregation of those local reversal flows forms separation lines. It should be emphasized in this high $R e_{c}$ case, we do not discern clear instantaneous separation of the turbulent flow or small-scale reversal flows along the flow direction compared with the previous lower Reynolds number cases.

\subsection{Comparisons with other turbulent flows}

It is interesting to compare the skin-friction portraits of airfoil flows to other canonical flows. When turbulent separation phenomenon takes place in turbulent boundary layer flow, as described by Chong et al. (1998), although a clear separation line cannot be observed, a region of small reversal flows is visible around the mean flow separation point. In flow past a circular cylinder, unsteady separation phenomena, especially the secondary separation/reattachment, dominate the mean features of the flow. For high Reynolds number cases, the unsteady secondary separation/reattachment can also be observed from instantaneous skin-friction portraits, revealed by the aggregation of small-scale reversal flows as noted in Cheng et al. (2017), while the spanwise distribution does not show much difference. This kind of effect is artificially enhanced 
in the grooved cylinder case of Cheng et al. (2018a), by using grooves distributed in the azimuthal direction while retaining the groove shape in the spanwise direction. Another case which also shows ordered small-scale reversal flows is that of a rotating cylinder as discussed in Cheng, Pullin \& Samtaney (2018b). In rotating cylinder, the lift crisis phenomenon, or so-called reverse Magnus effect, is found to be a result of flow reorganization due to the aggregation of small-scale separation/reattachment cells on the under and leeward parts of the cylinder surface. In this way, an ordered dividing line, which clearly shows upstream attached flow, and downstream small-scale reversal flows, is found to show little difference in the spanwise direction. This is quite similar to the turbulent separation in turbulent boundary layer flows.

However, in the present study of flow over airfoils, we note a stronger dependence or flow distortion in the spanwise distribution. In cases with $R e_{c}=10^{4}$ and $10^{5}$, along the flow evolution, we note the presence of local structures, with a variation in spanwise direction. Such structures show different intensities at different instants, for example, a strong deviation from separation point $S_{3}$ in figure 19 at around $x / C \approx 0.41$. Strong spanwise distribution is even obvious for the turbulent separation case at $R e_{c}=2.1 \times 10^{6}$. In this case, a clear dividing line that separates the incoming flow and small-scale reversal flows is hardly observed, which is unlike what is seen in a flat-plate turbulent boundary layer and cylinder flows.

In summary, airfoil flow shows quite similar but still exhibit unique behaviour compared to classical flows such as flow over a cylinder and turbulent boundary layer flow. In the case of $R e_{c}=10^{4}$, a primary separation bubble and a secondary separation bubble are clearly visible and their interaction forms the source of shear-layer turbulent transition, which is similar to the flow over a smooth cylinder. In the case of $R e_{c}=10^{5}$, the separation region breaks into several unsteady bubbles and their interaction results in strong spanwise variation and further evolution into turbulence. This strong spanwise structure provides a reference length scale for reasonably predicting flows. For the case $\operatorname{Re}_{c}=2.1 \times 10^{6}$, besides the similar turbulent transition behaviour, the turbulent separation phenomenon is similar to flat-plate turbulent separation in the sense of small-scale reversal flows, but still possesses its own character due to vortex shedding.

\section{Conclusion}

In this study, we have presented results of wall-modelled large-eddy simulations of flow past three different airfoils (NACA0012, NACA0018 and A-airfoil) at Reynolds number varying from $R e_{c}=10^{4}$ to $2.1 \times 10^{6}$. A Dirichlet boundary condition for the tangential velocity components is derived at a virtual wall in generalized curvilinear coordinates, which is coupled with an ordinary differential equation governing the wall shear stress (which is integrated analytically in time after some simplifying assumptions are made). We employ the stretched spiral vortex model for the SGS tensor in the computational domain. The numerical methodology is based on fourth-order spatial differencing, with a multigrid Poisson solver for the pressure utilizing Gauss-Seidel line smoothers. The formulation presented in generalized curvilinear coordinates opens up the possibility of using the proposed LES methodology with the associated wall models for a complex geometry.

At relative low Reynolds number $\left(R e_{c}=10^{4}\right)$ detailed comparisons (velocity profiles, skin friction and Reynolds stresses) between the wall-modelled LES (WMLES) and DNS show excellent agreement including the capturing of a near trailing edge separation bubble on the suction side of the NACA0012 airfoil. For the NACA0018 
airfoil case at moderately higher Reynolds number $\left(R e_{c}=10^{5}\right)$, comparisons with experiments of pressure coefficient, skin friction, average and r.m.s. velocity profiles show good agreement with the largest difference noted for the streamwise fluctuating velocity close to the reattachment point. For the A-airfoil, at even higher Reynolds number $\left(R e_{c}=2.1 \times 10^{6}\right)$, good agreement between WMLES and experiments is noted: the WMLES does not capture the tiny separation bubble near the leading edge after time and spanwise averaging although flow reversals in instantaneous velocity are noted; moreover, the WMLES Reynolds stresses show trends that are in agreement with experiments.

In the present study, we also examined the anisotropy of the flow in the context of the Lumley triangle. We note that all points lie within the triangle, as required by the realizability condition. For the low Reynolds number case, we note that in the near-wall region the turbulence approaches the two-component state progressing along the axisymmetric expansion line similar to the case of the log region in a channel flow. Within the separation zone, the behaviour is similar to that of free shear layers, as expected. The moderate Reynolds number case behaves similarly to the lower Reynolds number case, and once again several points lie on the log-law region. For the highest Reynolds number case, we see signatures of progress from $2 \mathrm{C}$ turbulence to log law to free shear-layer flow; although in this case the anisotropy in the free shear layer is considerably lower.

In this study, we examined the surface streamlines for three airfoils at the three different Reynolds numbers. For the low Reynolds number case, we noted that the surface streamlines remain approximately aligned with the streamwise direction over a significant portion of the airfoil surface before significant meandering in the spanwise direction is observed. As the Reynolds number is increased, the surface streamline plots show significant departures from the streamwise direction, with several secondary separation and reattachment points that are very unsteady compared with the nearly steady primary separation point. These unsteady secondary separation/reattachment bubbles were also noted in previous wall-resolved LES by Cheng et al. (2017) of flow past a circular cylinder. A reasonable conclusion is that the flow separation patterns show some coherence with separation/reattachment lines aligned along the spanwise direction before eventually becoming unsteady with no directional spanwise alignment in general for all cases.

\section{Acknowledgements}

The Cray XC40 Shaheen II at KAUST was used for all simulations reported. This research was partially supported under KAUST OCRF URF/1/1394-01 and under baseline research funds of R.S.

\section{Appendix A. Equation for $\langle q\rangle$}

From the definition of $\tilde{u}_{s},(3.10)$ and combining (3.8a) and (3.8b), we can obtain the streamwise momentum equation along the wall,

$$
\begin{aligned}
\frac{\partial \tilde{u}_{s}}{\partial t}= & \frac{\partial \tilde{u}}{\partial t} \cos \theta_{w}+\frac{\partial \tilde{v}}{\partial t} \sin \theta_{w} \\
= & -\frac{\cos \theta_{w}}{\sqrt{g}} \frac{\partial}{\partial \xi^{m}}\left(\widetilde{U^{m} u}+\sqrt{g} \frac{\partial \xi^{m}}{\partial x} \tilde{p}-v G^{m n} \frac{\partial \tilde{u}}{\partial \xi^{n}}\right) \\
& -\frac{\sin \theta_{w}}{\sqrt{g}} \frac{\partial}{\partial \xi^{m}}\left(\widetilde{U^{m} v}+\sqrt{g} \frac{\partial \xi^{m}}{\partial y} \tilde{p}-v G^{m n} \frac{\partial \tilde{v}}{\partial \xi^{n}}\right) .
\end{aligned}
$$


Then combining (3.8c), (3.15) and (A 1), we can obtain

$$
\frac{\partial\langle q\rangle}{\partial t}=F_{\xi}+F_{\eta}+F_{\zeta},
$$

where

$$
\begin{aligned}
& F_{\xi}=-\frac{1}{h} \int_{0}^{h} \frac{\tilde{u}_{s}}{\tilde{q}} \frac{\cos \theta_{w}}{\sqrt{g}} \frac{\partial}{\partial \xi^{1}}\left(\widetilde{U^{1} u}+\sqrt{g} \frac{\partial \xi^{1}}{\partial x} \tilde{p}-v G^{1 n} \frac{\partial \tilde{u}}{\partial \xi^{n}}\right) \mathrm{d} \eta \\
& -\frac{1}{h} \int_{0}^{h} \frac{\tilde{u}_{s}}{\tilde{q}} \frac{\sin \theta_{w}}{\sqrt{g}} \frac{\partial}{\partial \xi^{1}}\left(\widetilde{U^{1} v}+\sqrt{g} \frac{\partial \xi^{1}}{\partial y} \tilde{p}-v G^{1 n} \frac{\partial \tilde{v}}{\partial \xi^{n}}\right) \mathrm{d} \eta \\
& -\frac{1}{h} \int_{0}^{h} \frac{\tilde{w}}{\tilde{q}} \frac{1}{\sqrt{g}} \frac{\partial}{\partial \xi^{1}}\left(\widetilde{U^{1} w}+\sqrt{g} \frac{\partial \xi^{1}}{\partial z} \tilde{p}-v G^{1 n} \frac{\partial \tilde{w}}{\partial \xi^{n}}\right) \mathrm{d} \eta, \\
& F_{\eta}=-\frac{1}{h} \int_{0}^{h} \frac{\tilde{u}_{s}}{\tilde{q}} \frac{\cos \theta_{w}}{\sqrt{g}} \frac{\partial}{\partial \xi^{2}}\left(\widetilde{U^{2} u}+\sqrt{g} \frac{\partial \xi^{2}}{\partial x} \tilde{p}-v G^{2 n} \frac{\partial \tilde{u}}{\partial \xi^{n}}\right) \mathrm{d} \eta \\
& -\frac{1}{h} \int_{0}^{h} \frac{\tilde{u}_{s}}{\tilde{q}} \frac{\sin \theta_{w}}{\sqrt{g}} \frac{\partial}{\partial \xi^{2}}\left(\widetilde{U^{2} v}+\sqrt{g} \frac{\partial \xi^{2}}{\partial y} \tilde{p}-v G^{2 n} \frac{\partial \tilde{v}}{\partial \xi^{n}}\right) \mathrm{d} \eta \\
& -\frac{1}{h} \int_{0}^{h} \frac{\tilde{w}}{\tilde{q}} \frac{1}{\sqrt{g}} \frac{\partial}{\partial \xi^{2}}\left(\widetilde{U^{2} w}+\sqrt{g} \frac{\partial \xi^{2}}{\partial z} \tilde{p}-v G^{2 n} \frac{\partial \tilde{w}}{\partial \xi^{n}}\right) \mathrm{d} \eta, \\
& F_{\zeta}=-\frac{1}{h} \int_{0}^{h} \frac{\tilde{u}_{s}}{\tilde{q}} \frac{\cos \theta_{w}}{\sqrt{g}} \frac{\partial}{\partial \xi^{3}}\left(\widetilde{U^{3} u}+\sqrt{g} \frac{\partial \xi^{3}}{\partial x} \tilde{p}-v G^{3 n} \frac{\partial \tilde{u}}{\partial \xi^{n}}\right) \mathrm{d} \eta \\
& -\frac{1}{h} \int_{0}^{h} \frac{\tilde{u}_{s}}{\tilde{q}} \frac{\sin \theta_{w}}{\sqrt{g}} \frac{\partial}{\partial \xi^{3}}\left(\widetilde{U^{3} v}+\sqrt{g} \frac{\partial \xi^{3}}{\partial y} \tilde{p}-v G^{3 n} \frac{\partial \tilde{v}}{\partial \xi^{n}}\right) \mathrm{d} \eta \\
& -\frac{1}{h} \int_{0}^{h} \frac{\tilde{w}}{\tilde{q}} \frac{1}{\sqrt{g}} \frac{\partial}{\partial \xi^{3}}\left(\widetilde{U^{3} w}+\sqrt{g} \frac{\partial \xi^{3}}{\partial z} \tilde{p}-v G^{3 n} \frac{\partial \tilde{w}}{\partial \xi^{n}}\right) \mathrm{d} \eta \text {. }
\end{aligned}
$$

For the purpose of modelling, we make the following two approximations within the first grid cell $0 \leqslant \eta \leqslant h$ :

(i) the velocity angle $\theta$, i.e. $\tilde{u}_{s} / \tilde{q}$ and $\tilde{w} / \tilde{q}$, is constant;

(ii) the Jacobian of the transformation, i.e. $\sqrt{g}$, is constant.

Then we can reduce $F_{\eta}$ to be

$$
F_{\eta}=M+\left.\frac{\nu G^{22}}{\sqrt{g} h} \frac{\partial \tilde{q}}{\partial \eta}\right|_{h}-\frac{\nu G^{22}}{\sqrt{g} h} \eta_{0} .
$$

where

$$
\begin{aligned}
M= & -\left.\frac{1}{\sqrt{g} h}\left[\frac{\tilde{u}_{s}}{\tilde{q}}\left(\widetilde{U^{2} u} \cos \theta_{w}+\widetilde{U^{2} v} \sin \theta_{w}\right)+\frac{\tilde{w}}{\tilde{q}} \widetilde{U^{2} w}\right]\right|_{h} \\
& +\left.\frac{v G^{21}}{\sqrt{g} h}\left[\frac{\tilde{u}_{s}}{\tilde{q}}\left(\frac{\partial \tilde{u}}{\partial \xi^{1}} \cos \theta_{w}+\frac{\partial \tilde{v}}{\partial \xi^{1}} \sin \theta_{w}\right)+\frac{\tilde{w}}{\tilde{q}} \frac{\partial \tilde{w}}{\partial \xi^{1}}\right]\right|_{h} \\
& +\left.\frac{v G^{23}}{\sqrt{g} h}\left[\frac{\tilde{u}_{s}}{\tilde{q}}\left(\frac{\partial \tilde{u}}{\partial \xi^{3}} \cos \theta_{w}+\frac{\partial \tilde{v}}{\partial \xi^{3}} \sin \theta_{w}\right)+\frac{\tilde{w}}{\tilde{q}} \frac{\partial \tilde{w}}{\partial \xi^{3}}\right]\right|_{h}
\end{aligned}
$$



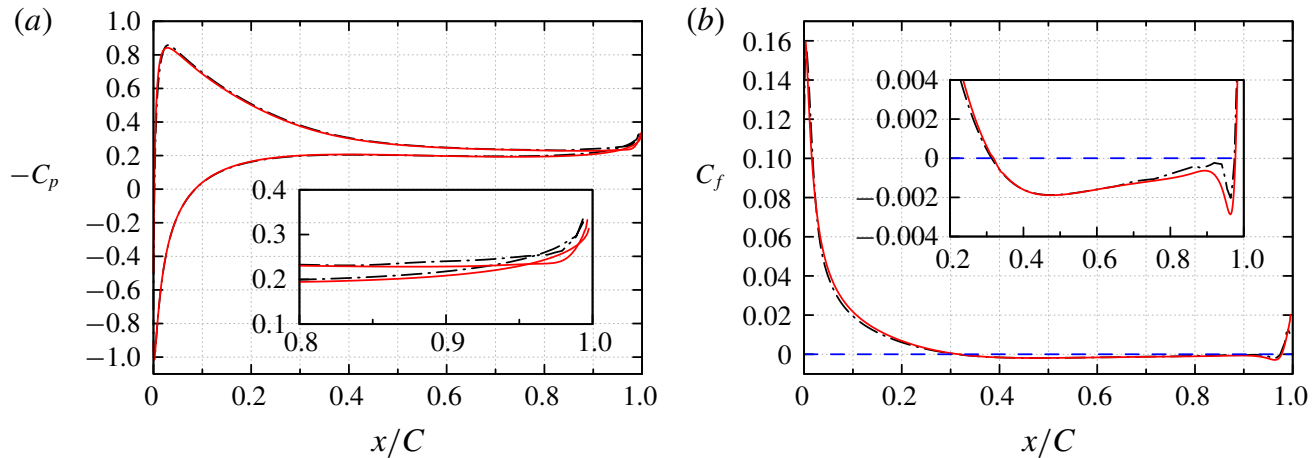

FIGURE 21. (Colour online) NACA0012, $R e_{c}=10^{4}$. (a) Distribution of the pressure coefficient $C_{p}$ around the airfoil, and $(b)$ skin-friction coefficient $C_{f}$ on the suction surface. - , WMLES with fine mesh; _ - _ , WMLES with coarse mesh. The - - - line $C_{f}=0$ is shown for convenience: zero crossing of this line indicates separation and reattachment.

If the first wall layer is forced to be orthogonal, then the terms with $G^{i j}(i \neq j)$ can be neglected, and $\eta$-direction is congruent with the $y_{n}$-direction, i.e. $\partial \tilde{q} /\left.\partial \eta\right|_{h}=\partial \tilde{q} /\left.\partial y_{n}\right|_{h}$. If the spanwise geometry is uniform, then the terms with $\partial \xi^{i} / \partial z(i=1,2)$ can be neglected.

\section{Appendix B. Analytical solution of the ODE for $\eta_{0}$}

The ODE for $\eta_{0},(3.16)$ can be rewritten as

$$
\frac{\partial \eta_{0}}{\partial t}=\left(C_{1}-C_{2} \eta_{0}\right) \eta_{0}
$$

and then

$$
\frac{\partial \eta_{0}}{\eta_{0}}+\frac{\partial \eta_{0}}{\frac{C_{1}}{C_{2}}-\eta_{0}}=C_{1} \partial t
$$

If $C_{1} / C_{2}$ is weakly dependent on $t$, (B 2) can be solved analytically,

$$
\eta_{0}(t)=\frac{C_{0} C_{1} e^{C_{1} \Delta t}}{C_{2}+C_{0} C_{2} e^{C_{1} \Delta t}},
$$

where

$$
C_{0}=\frac{\eta_{0}\left(t_{0}\right)}{\frac{C_{1}}{C_{2}}-\eta_{0}\left(t_{0}\right)}, \quad t=t_{0}+\Delta t
$$

and $t_{0}$ is the current time, $\Delta t$ is the time interval in the simulations.

\section{Appendix C. The effects of mesh resolution on WMLES}

To evaluate the effects of mesh resolution on WMLES, another WMLES with a coarser mesh $\left(N_{\xi} \times N_{\eta} \times N_{z}=512 \times 64 \times 32\right)$ is performed for the NACA0012 case 
$\left(R e_{c}=10^{4}, A o A=5^{\circ}, L_{z}=0.8 C\right)$. The wall-normal mesh size $\Delta \eta$ is doubled, and correspondingly the height of the virtual wall is doubled since we have fixed $h_{0}=$ $0.18 \Delta \eta$. We note that the maximum $\Delta \eta^{+}$still lies in the logarithmic region, but also that care must be exercised to have a decent resolution of the very thin laminar boundary layer near the leading edge for the subsequent flow development, such as separation and shear-layer transition (Park \& Moin 2014). To show convergence, in figure 21 we plot both the time- and spanwise-averaged pressure and skin-friction coefficients around the NACA0012 airfoil. It is evident from the $C_{p}$-plot that the peak value of WMLES with the coarse mesh is slightly larger than that with the fine mesh, as reported in $\S 5.1$. A larger discrepancy is observed at the trailing edge, i.e. close to the reattachment point. Both these two meshes work well for the prediction of separation and subsequent reattachment, although the absolute peak value of $C_{f}$ in the separation bubble of the coarse mesh WMLES is smaller than the fine mesh. Overall, these comparisons indicate that the present wall model can capture the separation bubble well even with the coarse mesh.

\section{REFERENCES}

Aввотt, I. H., Doenhoff, A. E. V. \& Stivers, J. L. 1945 Summary of airfoil data. NACA Tech. Rep. 824.

AsAdA, K. \& KAWAI, S. 2018 Large-eddy simulation of airfoil flow near stall condition at Reynolds number $2.1 \times 10^{6}$. Phys. Fluids 30 (8), 085103.

Asada, K., Sato, M., Nonomura, T., Kawai, S., Aono, H., Yakeno, A. \& Fujil, K. 2014 LES on turbulent separated flow around NACA0015 at Reynolds number 1600000 toward active flow control. In 32nd AIAA Applied Aerodynamics Conference, Atlanta, Georgia, AIAA.

Bae, H. J., Lozano-Durán, A., Bose, S. T. \& Moin, P. 2019 Dynamic slip wall model for large-eddy simulation. J. Fluid Mech. 859, 400-432.

Bell, J. H. \& Mehta, R. D. 1990 Development of a two-stream mixing layer from tripped and untripped boundary layers. AIAA J. 28 (12), 2034-2042.

Bose, S. T. \& MoIN, P. 2014 A dynamic slip boundary condition for wall-modeled large-eddy simulation. Phys. Fluids 26, 015104.

Bose, S. T. \& PARK, G. I. 2018 Wall-modeled large-eddy simulation for complex turbulent flows. Annu. Rev. Fluid Mech. 50, 535-561.

Boutilier, M. S. H. \& YARUSEvych, S. 2012 Separated shear layer transition over an airfoil at a low Reynolds number. Phys. Fluids 24, 084105.

Buchmannand, N. A., ATKInson, C. \& Soria, J. 2013 Influence of ZNMF jet flow control on the spatio-temporal flow structure over a NACA-0015 airfoil. Exp. Fluids 54 (3), 1485-1498.

САвот, W. \& Moin, P. 1999 Approximate wall boundary conditions in the large-eddy simulation of high Reynolds number flow. Flow Turbul. Combust. 63, 269-291.

Chaouat, B. 2006 Reynolds stress transport modeling for high-lift airfoil flows. AIAA J. 44 (10), 2390-2404.

Cheng, W., Pullin, D. I. \& Samtaney, R. 2015 Large-eddy simulation of separation and reattachment of a flat plate turbulent boundary layer. J. Fluid Mech. 785, 78-108.

Cheng, W., Pullin, D. I. \& Samtaney, R. 2018a Large-eddy simulation of flow over a grooved cylinder up to transcritical Reynolds numbers. J. Fluid Mech. 835, 327-362.

Cheng, W., Pullin, D. I. \& SAmtaney, R. $2018 b$ Large-eddy simulation of flow over a rotating cylinder: the lift crisis at $\operatorname{Re}_{D}=6 \times 10^{4}$. J. Fluid Mech. 855, 371-407.

Cheng, W., Pullin, D. I., Samtaney, R., Zhang, W. \& GaO, W. 2017 Large-eddy simulation of flow over a cylinder with $\operatorname{Re}_{D}$ from $3.9 \times 10^{3}$ to $8.5 \times 10^{5}$ : a skin-friction perspective. J. Fluid Mech. 820, 121-158.

Chol, H. \& MoIN, P. 2012 Grid-point requirements for large eddy simulation: Chapmans estimates revisited. Phys. Fluids 24, 011702. 
Choi, K. S. \& Lumley, J. L. 2001 The return to isotropy of homogeneous turbulence. J. Fluid Mech. 436, 59-84.

Chong, M. S., Soria, J., Perry, A. E., Chacin, J., Cantwell, B. J. \& Na, Y. 1998 Turbulence structures of wall-bounded shear flows found using DNS data. J. Fluid Mech. 357, 225-247.

Chung, D. \& Pullin, D. I. 2009 Large-eddy simulation and wall modelling of turbulent channel flow. J. Fluid Mech. 631, 281-309.

DAhlstrom, S. \& DAVIDSON, L. 2001 Large eddy simulation of the flow around an airfoil. In 39th Aerospace Sciences Meeting and Exhibit, Reno, Nevada, AIAA.

Diurno, G. V. 2001 Wall models for large-eddy simulation of non-equilibrium flows. PhD thesis, University of Maryland, College Park, MD.

Fröhlich, J., Mellen, C. P., Rodi, W., Temmerman, L. \& Leschziner, M. A. 2005 Highly resolved large-eddy simulation of separated flow in a channel with streamwise periodic constrictions. J. Fluid Mech. 526, 19-66.

George, K. J. \& Lele, S. K. 2014 Wall modeled large eddy simulation of airfoil trailing edge noise. In 20th AIAA/CEAS Aeroacoustics Conference, Atlanta, Georgia, AIAA.

Germano, M. 2004 Properties of the hybrid RANS/LES filter. Theor. Comput. Fluid Dyn. 17 (4), 225-231.

GLeYZES, C. 1988 Opération décrochage-résultats des essais à la soufflerie F2. Tech. Rep., RT-OA 19/5025, ONERA. Chatillon, France.

GRÖtZBACH, G. 1987 Direct numerical and large eddy simulation of turbulent channel flows. Encyclopedia of Fluid Mech. 6, 1337-1391.

Hosseini, S. M., Vinuesa, R., Schlatter, P., Hanifi, A. \& Henningson, D. S. 2016 Direct numerical simulation of the flow around a wing section at moderate Reynolds number. Intl $J$. Heat Fluid Flow 61, 117-128.

Inoue, M., Mathis, R., Marusic, I. \& Pullin, D. I. 2012 Inner-layer intensities for the flat-plate turbulent boundary layer combining a predictive wall-model with large-eddy simulations. Phys. Fluids 24, 075102.

InOUe, M. \& PUllin, D. I. 2011 Large-eddy simulation of the zero-pressure-gradient turbulent boundary layer up to $R e_{\theta}=O\left(10^{12}\right)$. J. Fluid Mech. 686, 507-533.

Inoue, M., Pullin, D. I., HARun, Z. \& MARusic, I. 2013 LES of the adverse-pressure gradient turbulent boundary layer. Intl J. Heat Fluid Flow 44, 293-300.

KAWAI, S. \& ASADA, K. 2013 Wall-modeled large-eddy simulation of high Reynolds number flow around an airfoil near stall condition. Comput. Fluids 85, 105-113.

KIRK, T. M. \& YARUSEVYCH, S. 2017 Vortex shedding within laminar separation bubbles forming over an airfoil. Exp. Fluids 58, 43-59.

Kitsios, V., Cordier, L., Bonnet, J.-P., OOI, A. \& Soria, J. 2011 On the coherent structures and stability properties of a leading-edge separated aerofoil with turbulent recirculation. J. Fluid Mech. 683, 395-416.

Laitone, E. V. 1997 Wind tunnel tests of wings at Reynolds numbers below 70000. Exp. Fluids 23 (5), 405-409.

Lissaman, P. B. S. 1983 Low-Reynolds-number airfoils. Annu. Rev. Fluid Mech. 15, 223-239.

Lumley, J. L. 1979 Computational modeling of turbulent flows. Adv. Appl. Mech. 18, 123-176.

Lumley, J. L. \& Newman, G. R. 1977 The return to isotropy of homogeneous turbulence. J. Fluid Mech. 82, 161-178.

Lundgren, T. S. 1982 Strained spiral vortex model for turbulent fine structure. Phys. Fluids 25 (12), 2193-2203.

Marusic, I., Kunkel, G. J. \& Porté-Agel, F. 2001 Experimental study of wall boundary conditions for large-eddy simulation. J. Fluid Mech. 446, 309-320.

MARY, I. \& SAgAUT, P. 2002 Large eddy simulation of flow around an airfoil near stall. AIAA J. 40 (6), 1139-1145.

Mellen, C. P., Frögrave, J. \& Rodi, W. 2003 Lessons from LESFOIL project on large-eddy simulation of flow around an airfoil. AIAA J. 41 (4), 573-581.

Misra, A. \& Pullin, D. I. 1997 A vortex-based subgrid stress model for large-eddy simulation. Phys. Fluids 9 (8), 2443-2454. 
Morgan, P. \& Visbal, M. 2003 Large-eddy simulation modeling issues for flow around wing sections. In 33rd AIAA Fluid Dynamics Conference and Exhibit, Orlando, Florida, AIAA.

Morinishi, Y., Lund, T. S., VAsilyev, O. V. \& Moin, P. 1998 Fully conservative higher order finite difference schemes for incompressible flow. J. Comput. Phys. 143, 90-124.

Nakano, T., Fujisawa, N. \& LeE, S. 2006 Measurement of tonal-noise characteristics and periodic flow structure around NACA0018 airfoil. Exp. Fluids 40 (3), 482-490.

PARK, G. I. \& MOIN, P. 2014 An improved dynamic non-equilibrium wall-model for large eddy simulation. Phys. Fluids 26, 015108.

PARK, G. I. \& MoIN, P. 2016 Numerical aspects and implementation of a two-layer zonal wall model for les of compressible turbulent flows on unstructured meshes. J. Comput. Phys. 305, 589-603.

Piomelli, U. 2008 Wall-layer models for large-eddy simulations. Prog. Aerosp. Sci. 44 (6), 437-446.

Piomelli, U. \& Balaras, E. 2002 Wall-layer models for large-eddy simulations. Annu. Rev. Fluid Mech. 34, 349-374.

Piomelli, U., Ferziger, J. H., Moin, P. \& Kim, J. 1989 New approximate boundary conditions for large eddy simulations of wall-bounded flows. Phys. Fluids A 1 (6), 1061-1068.

Pope, S. B. 2000 Turbulent Flows. Cambridge University Press.

SAito, N. \& Pullin, D. I. 2014 Large eddy simulation of smooth-rough-smooth transitions in turbulent channel flows. Intl J. Heat Mass Transfer 78, 707-720.

Saito, N., Pullin, D. I. \& InOUe, M. 2012 Large eddy simulation of smooth-wall, transitional and fully rough-wall channel flow. Phys. Fluids 24, 075103.

Sato, M., Asada, K., Nonomura, T., Kawai, S. \& FujiI, K. 2016 Large-eddy simulation of NACA 0015 airfoil flow at Reynolds number of $1.6 \times 10^{6}$. AIAA J. 55(2), 673-679.

Schmidt, S., Franke, M. \& Thiele, F. 2001 Assessment of SGS models in LES applied to a NACA 4412 airfoil. In 39th Aerospace Sciences Meeting and Exhibit, Reno, Nevada, AIAA.

Schmidt, S. \& Thiele, F. 2003 Detached eddy simulation of flow around A-airfoil. Flow Turbul. Combust. 71, 261-278.

SCHUMANN, U. 1975 Subgrid scale model for finite difference simulations of turbulent flows in plane channels and annuli. J. Comput. Phys. 18 (4), 376-404.

SRidhar, A., Pullin, D. I. \& Cheng, W. 2017 Rough-wall turbulent boundary layers with constant skin friction. J. Fluid Mech. 818, 26-45.

Townsend, A. A. 1976 The Structure of Turbulent Shear Flow. Cambridge University Press.

VoelKL, T., Pullin, D. I. \& ChAN, D. C. 2000 A physical-space version of the stretched-vortex subgrid-stress model for large-eddy simulation. Phys. Fluids 12 (7), 1810-1825.

Yarusevych, S., Sullivan, P. E. \& Kawall, J. G. 2006 Coherent structures in an airfoil boundary layer and wake at low Reynolds numbers. Phys. Fluids 18, 044101.

Yarusevych, S., Sullivan, P. E. \& Kawall, J. G. 2009 On vortex shedding from an airfoil in low-Reynolds-number flows. J. Fluid Mech. 632, 245-271.

ZANG, T. A. 1991 On the rotation and skew-symmetric forms for incompressible flow simulations. Appl. Numer. Maths 7, 27-40.

Zang, Y., Street, R. L. \& KosefF, J. R. 1994 A non-staggered grid, fractional step method for time-dependent incompressible Navier-Stokes equations in curvilinear coordinates. J. Comput. Phys. 114, 18-33.

Zhang, W., Cheng, W., Gao, W., Qamar, A. \& Samtaney, R. 2015 Geometrical effects on the airfoil flow separation and transition. Comput. Fluids 116, 60-73.

ZhANG, W. \& SAMTANEY, R. 2016 Assessment of spanwise domain size effect on the transitional flow past an airfoil. Comput. Fluids 124, 39-53.

Zhou, Y. \& WANG, Z. J. 2012 Effects of surface roughness on separated and transitional flows over a wing. AIAA J. 50 (3), 593-609. 